\title{
LOWER SEMICONTINUITY AND RELAXATION RESULTS IN BV FOR INTEGRAL FUNCTIONALS WITH BV INTEGRANDS
}

\author{
Micol Amar ${ }^{1}$, Virginia De CiCCO $^{1}$ And Nicola Fusco ${ }^{2}$
}

\begin{abstract}
New $L^{1}$-lower semicontinuity and relaxation results for integral functionals defined in $\mathrm{BV}(\Omega)$ are proved, under a very weak dependence of the integrand with respect to the spatial variable $x$. More precisely, only the lower semicontinuity in the sense of the 1-capacity is assumed in order to obtain the lower semicontinuity of the functional. This condition is satisfied, for instance, by the lower approximate limit of the integrand, if it is BV with respect to $x$. Under this further BV dependence, a representation formula for the relaxed functional is also obtained.
\end{abstract}

Mathematics Subject Classification. 49J45, 49Q20, 49M20.

Received October 20, 2006. Revised January 11, 2007.

Published online November 21, 2007.

\section{INTRODUCTION}

In this paper we study the $L^{1}$-lower semicontinuity and relaxation of an integral functional of the type

$$
F(u)=\int_{\Omega} f(x, u(x), \nabla u(x)) \mathrm{d} x,
$$

where $u$ is a scalar function from $W^{1,1}(\Omega)$, and of its BV counterpart

$$
\mathcal{F}(u)=\int_{\Omega} f(x, u, \nabla u) \mathrm{d} x+\int_{\Omega} f^{\infty}\left(x, \widetilde{u}, \frac{D^{c} u}{\left|D^{c} u\right|}\right) \mathrm{d}\left|D^{c} u\right|+\int_{J_{u} \cap \Omega} \mathrm{d} \mathcal{H}^{N-1} \int_{u^{-}(x)}^{u^{+}(x)} f^{\infty}\left(x, s, \nu_{u}(x)\right) \mathrm{d} s .
$$

In recent years there has been a renewed interest in these topics since many authors $[2,3,13-15,19,22-26]$ have studied the lower semicontinuity and relaxation of $F$ and $\mathcal{F}$ with the aim of lessening the regularity assumptions on $f$ with respect to $x$.

In searching weaker conditions on $f$ which still guarantee the lower semicontinuity essentially two kinds of assumptions are considered in the above papers (beside the usual requirements that $f(x, s, \xi)$ is convex in $\xi$ and

\footnotetext{
Keywords and phrases. Semicontinuity, relaxation, BV functions, capacity.

1 Dipartimento di Metodi e Modelli Matematici, Università di Roma "La Sapienza", Via A. Scarpa 16, 00161 Roma, Italy; amar@dmmm.uniroma1.it; decicco@dmmm.uniroma1.it

2 Dipartimento di Matematica e Applicazioni, Università di Napoli "Federico II", Complesso di Monte Sant'Angelo, Via Cintia, 80126 Napoli, Italy; nicola.fusco@unina.it
} 
continuous in $s$ ). Either $f$ is assumed to be lower semicontinuous in $x$, uniformly with respect to $(s, \xi)$ (see $e . g$. $[19,23])$, or $f$ is assumed to be weakly differentiable (see $[13,14,22,25]$ ) or even BV in $x$ (see [15]).

In this paper we address the lower semicontinuity and relaxation issues under different assumptions on $f$. To illustrate them let us consider the model case where $f(x, \xi)=a(x) p(\xi)$ and $p: \mathbb{R}^{N} \rightarrow[0,+\infty)$ is a convex function. As a consequence of an approximation result due to Dal Maso (see [11]) and of a recent lower semicontinuity theorem proved in [14], we obtain that if $a: \Omega \rightarrow[0,+\infty)$ is lower semicontinuous in the sense of 1-capacity (see Sect. 2.3), then $\mathcal{F}$ is $L^{1}$-lower semicontinuous in $\mathrm{BV}(\Omega)$. As far as we know, this is the first result of this kind where capacity plays a role as an assumption on the integrand. Moreover, Theorem 3.1 is an extension of the result proved in [19], which requires $a$ to be lower semicontinuity in the classical sense. Notice also that if $a$ is a BV function, then it can be proved that its lower approximate limit $a^{-}$is lower semicontinuous in the sense of 1-capacity. Thus our result implies immediately those proved for instance in [14,25] or in [15] where $a$ is assumed to be in $W^{1,1}(\Omega)$ or in $\mathrm{BV}(\Omega)$, respectively.

The assumption that $a$ is a BV function is also a key point in finding the relaxed functional with respect to $L^{1}$-convergence of

$$
u \in W^{1,1}(\Omega) \mapsto \int_{\Omega} a(x) p(\nabla u(x)) \mathrm{d} x .
$$

In fact, as stated in the first part of Theorem 1.1 below, we have that if $a \in \operatorname{BV}(\Omega)$, the relaxed functional of $(1.1)$ in $\operatorname{BV}(\Omega)$ is given by

$$
\int_{\Omega} a(x) p(\nabla u) \mathrm{d} x+\int_{\Omega} a^{-}(x) p^{\infty}\left(\frac{D^{c} u}{\left|D^{c} u\right|}\right) \mathrm{d}\left|D^{c} u\right|+\int_{J_{u} \cap \Omega}\left(u^{+}-u^{-}\right) a^{-}(x) p^{\infty}\left(\nu_{u}(x)\right) \mathrm{d} \mathcal{H}^{N-1} .
$$

Notice that the lower semicontinuity result and the representation formula for the relaxed functional of (1.1) are obtained under different assumptions on $a$. This is not the case when dealing with one dimensional functionals. In fact, it can be proved as a consequence of Example 2 in [6] that, given any Borel function $a$, the functional

$$
\int_{\Omega} a(x) p\left(u^{\prime}\right) \mathrm{d} x+\int_{\Omega} a(x) p^{\infty}\left(\frac{D^{c} u}{\left|D^{c} u\right|}\right) \mathrm{d}\left|D^{c} u\right|+\sum_{x \in J_{u} \cap \Omega} a(x) p^{\infty}(u(x+)-u(x-))
$$

is $L^{1}$-lower semicontinuous on $\mathrm{BV}$ if and only if $a$ is lower semicontinuous and that the relaxed functional of (1.1) is represented by (1.3) with $a$ replaced by its lower semicontinuous envelope.

However, in higher dimension things are more complicate. Indeed, examples can be given of functionals of the type (1.1) whose relaxed functional is given by a functional of the type $\mathcal{F}$ where the integrand $f$ is not the product of two functions of $x$ and $\xi$ (see [1], Sect. 8).

In the case of a general integrand $f$ our Theorem 3.4 states that a (uniform) lower semicontinuity of $f$ in the sense of 1-capacity is still sufficient for the functional $\mathcal{F}$ to be lower semicontinuous. This result is obtained by combining in a delicate way two different approximation techniques: the first one was developed in [11], Section 1, in the context of capacity theory and the second one was introduced in [23] to approximate lower semicontinuous integrands, convex in the last variable.

On the other side, as in the model case, the BV dependence of $f$ with respect to $x$ turns out to guarantee that the relaxed functional $\bar{F}$ of $F$ is less than or equal to $\mathcal{F}$, provided that we choose $\left(f^{\infty}\right)^{-}$as representative of $f^{\infty}$ in $\mathcal{F}$. Notice in fact that the values of $\mathcal{F}$ are clearly affected by the choice of such representative. We remark that this choice makes the proof of inequality $\bar{F} \leq \mathcal{F}$ quite difficult. In fact, up to now, this inequality has been always proved under the assumption that $f^{\infty}$ were upper semicontinuous with respect to $x$ (see $e . g$. $[2,3,19])$.

The inequality $\bar{F} \leq \mathcal{F}$ is proved in Theorem 4.2 , where the case of an integrand $f$ depending only on $(x, \xi)$ is considered. Even in this case the proof of this inequality requires a very delicate adaptation of the blow-up argument of Fonseca-Müller [20,21]. In fact the situation studied here is complicated by the interaction between the jump set of $u$ and the jump sets of the BV functions $f^{\infty}(\cdot, \xi)$ as $\xi$ varies in $\mathbb{R}^{N}$. Dealing with this difficulty 
requires some new technical ideas (see the discussion before Prop. 4.4), which at the moment do not seem to work when also a dependence on $s$ is allowed.

Combining the inequality $\bar{F} \leq \mathcal{F}$ with the lower semicontinuity result given in Theorem 3.4 one then gets that $\mathcal{F}$ coincides with $\bar{F}$.

The following relaxation result is a consequence of Theorems 3.6 and 4.2. This is not the most general one which can be derived by the combinations of these two theorems, but still covers some significant and interesting examples which were not included in the relaxation results so far available in the literature.

Theorem 1.1. Let $f: \Omega \times \mathbb{R}^{N} \rightarrow[0,+\infty)$ be a Borel function such that $f(x, \cdot)$ is convex for every $x \in \Omega$ and, for every $\xi \in \mathbb{R}^{N}, f(\cdot, \xi),\left(f^{-}\right)^{\infty}(\cdot, \xi)$ belong to $\mathrm{BV}(\Omega)$. Moreover, assume that there exists $\Lambda>0$ such that

$$
0 \leq f(x, \xi) \leq \Lambda(1+|\xi|) \quad \text { for all }(x, \xi) \in \Omega \times \mathbb{R}^{N},
$$

and that at least one of the following three conditions holds:

$f(x, \xi)$ can be splitted as a product of two functions depending on $x$ and $\xi$ separately;

$$
\left\{\begin{array}{l}
f(x, \cdot) \text { is positively } 1 \text {-homogeneous and } \\
f^{-}(x, \xi)>0 \text { for all }(x, \xi) \in\left(\Omega \backslash N_{0}\right) \times\left(\mathbb{R}^{N} \backslash\{0\}\right) \text {, where } \mathcal{H}^{N-1}\left(N_{0}\right)=0 .
\end{array}\right.
$$

$$
\left\{\begin{array}{l}
\text { There exists a convex and demicoercive function } \Psi: \mathbb{R}^{N} \rightarrow[0,+\infty) \text { such that } \\
\Psi(\xi) \leq f(x, \xi) \quad \text { for all }(x, \xi) \in \Omega \times \mathbb{R}^{N} ; \\
\text { for every } \xi \in \mathbb{R}^{N} \text { there exists } N_{\xi} \subset \Omega \text { such that } \mathcal{H}^{N-1}\left(N_{\xi}\right)=0 \text { and } \\
\left(f^{-}\right)^{\infty}(x, \xi)=\left(\left(f^{-}\right)^{\infty}\right)^{-}(x, \xi) \quad \text { for all } x \in \Omega \backslash N_{\xi} .
\end{array}\right.
$$

Then, the relaxed functional $\bar{F}(u)$ is given by

$$
\int_{\Omega} f(x, \nabla u) \mathrm{d} x+\int_{\Omega}\left(f^{-}\right)^{\infty}\left(x, \frac{D^{c} u}{\left|D^{c} u\right|}\right) \mathrm{d}\left|D^{c} u\right|+\int_{J_{u} \cap \Omega}\left[u^{+}(x)-u^{-}(x)\right]\left(f^{-}\right)^{\infty}\left(x, \nu_{u}(x)\right) \mathrm{d} \mathcal{H}^{N-1} .
$$

A few remarks are in order. First, observe that if $f(x, \xi)=a(x) p(\xi)$, the assumptions of the above theorem are clearly satisfied whenever $a$ is a bounded function in $\mathrm{BV}(\Omega)$ and $p$ is a convex function with linear growth, such that $f \geq 0$. Similarly, if $(1.5)$ is in force, then also $f^{-}(x, \cdot)$ is 1-homogeneous for all $x$, therefore in order to apply Theorem 1.1 it is enough to assume that $f(\cdot, \xi) \in \mathrm{BV}(\Omega)$ for all $\xi$. Notice also that the representation formula (1.7) for the relaxed functional $\bar{F}(u)$ reduces, in the first case, to (1.2) and, in the second case, to

$$
\int_{\Omega} f(x, \nabla u) \mathrm{d} x+\int_{\Omega} f^{-}\left(x, \frac{D^{c} u}{\left|D^{c} u\right|}\right) \mathrm{d}\left|D^{c} u\right|+\int_{J_{u} \cap \Omega}\left[u^{+}(x)-u^{-}(x)\right] f^{-}\left(x, \nu_{u}(x)\right) \mathrm{d} \mathcal{H}^{N-1} .
$$

On the other hand, for a general integrand it is not necessarily true that $\left(f^{-}\right)^{\infty}(\cdot, \xi)$ and $\left(\left(f^{-}\right)^{\infty}\right)^{-}(\cdot, \xi)$ coincide $\mathcal{H}^{N-1}$-a.e. in $\Omega$, as shown in [10], Example 4.4. In that case functional (1.7) is still lower semicontinuous in $\operatorname{BV}(\Omega)$ but it is strictly smaller than $\bar{F}(u)$, which in turn is represented by a similar formula, with $\left(f^{-}\right)^{\infty}$ replaced by $\left(\left(f^{-}\right)^{\infty}\right)^{-}$.

\section{Notation AND PRELIMinaRies}

\subsection{Notation}

Throughout the paper $N>1$ is a fixed integer and the letter $c$ denotes a strictly positive constant, whose value may change from line to line. 
Given $x_{0} \in \mathbb{R}^{N}$ and $\rho>0, B_{\rho}\left(x_{0}\right)$ denotes the ball in $\mathbb{R}^{N}$ centered in $x_{0}$ with radius $\rho$.

Let $\Omega$ be an open subset of $\mathbb{R}^{N}$. We denote by $\mathcal{A}(\Omega)$ the family of all open subsets $A$ of $\Omega$ and by $\mathcal{B}(\Omega)$ the $\sigma$-algebra of all Borel subsets $B$ of $\Omega$.

Set $\mathcal{L}^{N}$ the Lebesgue measure on $\mathbb{R}^{N}$ and $\mathcal{H}^{N-1}$ the Hausdorff measure of dimension $(N-1)$ on $\mathbb{R}^{N}$.

If $g \in L_{\mathrm{loc}}^{1}(\Omega)$ and $x \in \Omega$, the precise representative of $g$ at $x$ is defined as the unique value $\widetilde{g}(x) \in \mathbb{R}$ such that

$$
\lim _{\varrho \rightarrow 0^{+}} \frac{1}{\varrho^{N}} \int_{B_{\varrho}(x)}|g(y)-\widetilde{g}(x)| \mathrm{d} x=0 .
$$

The set of points in $\Omega$ where the precise representative of $x$ is not defined is called the approximate singular set of $g$ and denoted by $S_{g}$. If $g(x)=\widetilde{g}(x)$, we say that $g$ is approximately continuous at $x$.

The space $\mathrm{BV}(\Omega)$ is defined as the space of those functions $g: \Omega \rightarrow \mathbb{R}$ belonging to $L^{1}(\Omega)$ whose distributional gradient $D g$ is an $\mathbb{R}^{N}$-valued Radon measure with finite total variation $|D g|(\Omega)$.

We recall the usual decomposition

$$
D g=\nabla g \mathcal{L}^{N}+D^{c} g+\left(g^{+}-g^{-}\right) \nu_{g} \mathcal{H}^{N-1}\left\lfloor J_{g},\right.
$$

where $\nabla g$ is the Radon-Nikodým derivative of $D g$ with respect to the Lebesgue measure and $D^{c} g$ is the Cantor part of $D g$. Moreover, for $\mathcal{H}^{N-1}$-a.e. $x_{0} \in S_{g}$ there exist a unit vector $\nu_{g}\left(x_{0}\right)$ and two numbers $g^{-}\left(x_{0}\right)<g^{+}\left(x_{0}\right)$ such that

$$
\lim _{\varrho \rightarrow 0^{+}} \frac{1}{\varrho^{N}} \int_{B_{\varrho}^{ \pm}\left(x_{0}, \nu_{g}\left(x_{0}\right)\right)}\left|g(x)-g^{ \pm}\left(x_{0}\right)\right| \mathrm{d} x=0,
$$

where, for any $\nu \in \mathbb{S}^{N-1}, B_{\varrho}^{ \pm}\left(x_{0}, \nu\right)=\left\{x \in B_{\varrho}\left(x_{0}\right):\left\langle x-x_{0}, \nu\right\rangle \gtrless 0\right\}$. The set of points $x \in S_{g}$ where $-\infty<g^{-}(x)<g^{+}(x)<+\infty$ and (2.2) holds is called the jump set of $g$ and it is denoted by $J_{g}$.

For a general survey on BV functions we refer to [4].

\subsection{Slicing of BV functions. Traces}

Given a direction $\nu \in \mathbb{S}^{N-1}$, every point $x \in \mathbb{R}^{N}$ can be decomposed as $x=\left(x_{\nu}^{\perp}, x_{\nu}\right)$, with $x_{\nu}=\langle x, \nu\rangle$ and $x_{\nu}^{\perp}=x-x_{\nu} \nu$. By $\pi_{\nu^{\perp}}$ we denote the projection of $\mathbb{R}^{N}$ onto the plane through the origin orthogonal to $\nu$. If $E$ is a given subset of $\mathbb{R}^{N}$, we set $E_{x_{\nu}^{\perp}}=\left\{x_{\nu} \in \mathbb{R}:\left(x_{\nu}^{\perp}, x_{\nu}\right) \in E\right\}$. Similarly, if $g: \mathbb{R}^{N} \rightarrow \mathbb{R}$ is a given function, for every $x_{\nu}^{\perp} \in \mathbb{R}^{N-1}$, we denote by $g_{x_{\nu}}$ the restriction of the function $g$ to $\mathbb{R} ;$ i.e., the function $x_{\nu} \in \mathbb{R} \mapsto g\left(x_{\nu}^{\perp}, x_{\nu}\right)$.

Lemma 2.1 (see [4], Th. 3.108). Let $g \in \mathrm{BV}(\Omega)$ be a given function and $\nu \in \mathbb{S}^{N-1}$ be a given direction. Then, for $\mathcal{H}^{N-1}$-almost every $x_{\nu}^{\perp} \in \pi_{\nu^{\perp}}(\Omega), g_{x_{\nu}^{\perp}}$ belongs to $\mathrm{BV}\left(\Omega_{x_{\nu}^{\perp}}\right)$,

$$
J_{g_{x_{\nu}}}=\left(J_{g}\right)_{x_{\nu}^{\perp}}
$$

$(\widetilde{g})_{x_{\nu}^{\perp}}$ is continuous in $\Omega \backslash\left(J_{g}\right)_{x_{\nu}}$ and $\left\langle\nu, \nu_{g}\left(x_{\nu}^{\perp}, x_{\nu}\right)\right\rangle \neq 0$ for every $x_{\nu} \in\left(J_{g}\right)_{x_{\nu}^{\perp}}$. Moreover, for any $x_{\nu} \in\left(J_{g}\right)_{x_{\nu}^{\perp}}$,

$$
\begin{array}{ll}
g_{x_{\nu}^{\perp}}^{ \pm}\left(x_{\nu}\right)=\lim _{z_{\nu} \rightarrow x_{\nu} \pm} \widetilde{g}\left(x_{\nu}^{\perp}, z_{\nu}\right) & \text { if }\left\langle\nu, \nu_{g}\left(x_{\nu}^{\perp}, x_{\nu}\right)\right\rangle>0, \\
g_{x_{\nu}^{\perp}}^{ \pm}\left(x_{\nu}\right)=\lim _{z_{\nu} \rightarrow x_{\nu} \mp} \widetilde{g}\left(x_{\nu}^{\perp}, z_{\nu}\right) & \text { if }\left\langle\nu, \nu_{g}\left(x_{\nu}^{\perp}, x_{\nu}\right)\right\rangle<0 .
\end{array}
$$

Let $\Gamma$ be a $(N-1)$-dimensional manifold of class $\mathcal{C}^{1}$ in $\mathbb{R}^{N}$, oriented by a map $\nu_{\Gamma}: \Gamma \rightarrow \mathbb{S}^{N-1}$. Next theorem, which actually holds in a much more general setting (see, e.g. [4], Ths. 3.77 and 3.86), states that any BV function defined in a neighborhood of $\Gamma$ has traces on both sides of $\Gamma$. 
Theorem 2.2. Let $\Omega$ be an open set and $\Gamma \subset \Omega$ a $\mathcal{C}^{1}$ manifold oriented by $\nu_{\Gamma}$. Then, if $g \in \mathrm{BV}(\Omega)$, there exist $g_{\Gamma}^{ \pm} \in L^{1}\left(\Gamma, \mathcal{H}^{N-1}\right)$ such that, for $\mathcal{H}^{N-1}$-a.e. $x_{0} \in \Gamma$,

$$
\lim _{\varrho \rightarrow 0^{+}} \frac{1}{\varrho^{N}} \int_{B_{\varrho}^{ \pm}\left(x_{0}, \nu_{\Gamma}\left(x_{0}\right)\right)}\left|g(x)-g_{\Gamma}^{ \pm}\left(x_{0}\right)\right| \mathrm{d} x=0 .
$$

Remark 2.3. Notice that, since $g_{\Gamma}^{ \pm} \in L^{1}\left(\Gamma, \mathcal{H}^{N-1}\right)$, then $\mathcal{H}^{N-1}$-a.e. $x_{0} \in \Gamma$ is a Lebesgue point for $g_{\Gamma}^{ \pm}$. Therefore, for such a point we have

$$
\lim _{\varrho \rightarrow 0} \frac{1}{\varrho^{N-1}} \int_{\Gamma \cap B_{\varrho}\left(x_{0}\right)}\left|g_{\Gamma}^{ \pm}(x)-g_{\Gamma}^{ \pm}\left(x_{0}\right)\right| \mathrm{d} \mathcal{H}^{N-1}(x)=0 .
$$

Remark 2.4. Notice that from definition (2.1) and Theorem 2.2 it follows immediately that $g_{\Gamma}^{ \pm}(x)=\widetilde{g}(x)$ for $\mathcal{H}^{N-1}$-a.e. $x \in \Gamma \backslash J_{g}$. Moreover, since $\nu_{\Gamma}(x)= \pm \nu_{g}(x)$ for $\mathcal{H}^{N-1}$-a.e. $x \in \Gamma \cap J_{g}$ (see [4], Th. 3.78 and Rem. 2.87), from (2.2) and (2.3) we have $g_{\Gamma}^{ \pm}(x)=g^{ \pm}(x)$ for $\mathcal{H}^{N-1}$-a.e. $x \in \Gamma \cap J_{g}$ such that $\nu_{\Gamma}(x)=\nu_{g}(x)$ and $g_{\Gamma}^{ \pm}(x)=g^{\mp}(x)$ if $\nu_{\Gamma}(x)=-\nu_{g}(x)$.

Let us assume that the manifold $\Gamma$ splits $\Omega$ in two disjoint open subsets $\Omega^{ \pm}$and, just to fix the ideas, that $\nu_{\Gamma}$ points toward $\Omega^{+}$. Then, for every point $x_{0} \in \Gamma$ for which (2.3) holds, we have (see [4], Rem. 3.85):

$$
\lim _{\varrho \rightarrow 0^{+}} \frac{1}{\varrho^{N}} \int_{\Omega^{ \pm} \cap B_{\varrho}\left(x_{0}\right)}\left|g(x)-g_{\Gamma}^{ \pm}\left(x_{0}\right)\right| \mathrm{d} x=0 .
$$

\subsection{Capacity}

Given an open set $A \subset \mathbb{R}^{N}$, the 1-capacity of $A$ is defined by setting

$$
C_{1}(A):=\inf \left\{\int_{\mathbb{R}^{N}}|D \varphi| \mathrm{d} x: \varphi \in W^{1,1}\left(\mathbb{R}^{N}\right), \quad \varphi \geq 1 \quad \mathcal{L}^{N} \text {-a.e. on } A\right\} .
$$

Then, the 1-capacity of an arbitrary set $B \subset \mathbb{R}^{N}$ is given by

$$
C_{1}(B):=\inf \left\{C_{1}(A): A \supseteq B, U \text { open }\right\} .
$$

It is well known that capacities and Hausdorff measure are closely related. In particular, we have that for every Borel set $B \subset \mathbb{R}^{N}$

$$
C_{1}(B)=0 \quad \Longleftrightarrow \quad \mathcal{H}^{N-1}(B)=0 .
$$

Definition 2.5. Let $B \subset \mathbb{R}^{N}$ be a Borel set with $C_{1}(B)<+\infty$. Given $\varepsilon>0$, we call capacitary $\varepsilon$-quasi-potential (or simply capacitary quasi-potential) of $B$ a function $\varphi_{\varepsilon} \in W^{1,1}\left(\mathbb{R}^{N}\right)$, such that $0 \leq \widetilde{\varphi}_{\varepsilon} \leq 1 \mathcal{H}^{N-1}$-a.e. in $\mathbb{R}^{N}$, $\widetilde{\varphi}_{\varepsilon}=1 \mathcal{H}^{N-1}$-a.e. in $B$ and

$$
\int_{\mathbb{R}^{N}}\left|D \varphi_{\varepsilon}\right| \mathrm{d} x \leq C_{1}(B)+\varepsilon .
$$

We recall that a function $g: \mathbb{R}^{N} \rightarrow \mathbb{R}$ is said $C_{1}$-quasi continuous if for every $\varepsilon>0$ there exists an open set $A$, with $C_{1}(A)<\varepsilon$, such that $\left.g\right|_{A^{c}}$ is continuous on $A^{c} ; C_{1}$-quasi lower semicontinuous and $C_{1}$-quasi upper semicontinuous functions are defined similarly.

It is well known that if $g$ is a $W^{1,1}$-function, then its precise representative $\widetilde{g}$ is $C_{1}$-quasi continuous (see [18], Sects. 9 and 10). Moreover, to every BV-function $g$, it is possible to associate a $C_{1}$-quasi lower semicontinuous and a $C_{1}$-quasi upper semicontinuous representative, as stated by the following theorem (see [9], Th. 2.5).

Theorem 2.6. For every function $g \in \mathrm{BV}(\Omega)$, the approximate upper limit $g^{+}$and the approximate lower limit $g^{-}$are $C_{1}$-quasi upper semicontinuous and $C_{1}$-quasi lower semicontinuous, respectively. 
In particular, if $B$ is a Borel subset of $\mathbb{R}^{N}$ with finite perimeter, then $\chi_{B}^{-}$is $C_{1}$-quasi lower semicontinuous and $\chi_{B}^{+}$is $C_{1}$-quasi upper semicontinuous.

Finally we recall the following approximation result, due to Dal Maso (see [11], Lem. 1.5 and Sect. 6).

Lemma 2.7. Let $g: \mathbb{R}^{N} \rightarrow[0,+\infty)$ be a $C_{1}$-quasi lower semicontinuous function. Then there exists an increasing sequence of nonnegative functions $\left\{g_{h}\right\} \subseteq W^{1,1}\left(\mathbb{R}^{N}\right)$ such that, for every $h \in \mathbb{N}, g_{h}$ is approximately continuous $\mathcal{H}^{N-1}$-almost everywhere in $\mathbb{R}^{N}$ and $g_{h}(x) \rightarrow g(x)$, when $h \rightarrow+\infty$, for $\mathcal{H}^{N-1}$-almost every $x \in \mathbb{R}^{N}$.

\subsection{Demicoercive functions}

Definition 2.8. We say that a function $g: \mathbb{R}^{N} \rightarrow[0,+\infty)$ is demicoercive if there exist a vector $v \in \mathbb{R}^{N}$ and two constants $a>0, b \geq 0$ such that

$$
a|\xi| \leq g(\xi)+\langle v, \xi\rangle+b \quad \text { for all } \xi \in \mathbb{R}^{N} .
$$

It is not difficult to check that coercive or strictly convex functions are demicoercive. Moreover, if $g$ satisfies

$$
\lim _{|\xi| \rightarrow+\infty} g(\xi)=+\infty
$$

then it is demicoercive, too.

For other properties of demicoercive functions see $[5,24,26]$.

\subsection{The integrand $f$ and its recession function}

Let $f: \Omega \times \mathbb{R} \times \mathbb{R}^{N} \rightarrow[0,+\infty)$ be a Borel function. If $f$ is convex with respect to the last variable, the recession function of $f$ is defined for all $(x, s, \xi) \in \Omega \times \mathbb{R} \times \mathbb{R}^{N}$ by setting

$$
f^{\infty}(x, s, \xi)=\lim _{t \rightarrow+\infty} \frac{f(x, s, t \xi)}{t}=\sup _{t>0} \frac{f(x, s, t \xi)-f(x, s, 0)}{t} .
$$

Notice that, since the function $t \mapsto \frac{f(x, s, t \xi)-f(x, s, 0)}{t}$ is increasing, the above limit always exists. Moreover it is easily checked that $f^{\infty}$ is a Borel function, positively 1-homogeneous and convex in the last variable, and that

$$
\frac{f(x, s, t \xi)}{t} \leq f^{\infty}(x, s, \xi)+\frac{f(x, s, 0)}{t} \quad \text { for all } t>0 .
$$

In the sequel, we shall often assume that the Borel function $f$ satisfies the following conditions

$$
\begin{aligned}
& f(x, s, \cdot) \quad \text { is convex for every }(x, s) \in \Omega \times \mathbb{R} \\
& 0 \leq f(x, s, \xi) \leq \Lambda(1+|\xi|) \quad \text { for every }(x, s, \xi) \in \Omega \times \mathbb{R} \times \mathbb{R}^{N}
\end{aligned}
$$

for some positive constant $\Lambda$. Note that (2.7) and (2.8) imply that $f$ is Lipschitz continuous in the last variable, uniformly with respect to $(x, s) \in \Omega \times \mathbb{R}$. Moreover, from these assumptions, it follows that

$$
0 \leq f^{\infty}(x, s, \xi) \leq \Lambda|\xi| \quad \text { for every }(x, s, \xi) \in \Omega \times \mathbb{R} \times \mathbb{R}^{N} .
$$

Finally, since $f^{\infty}$ is convex with respect to $\xi$, by (2.9) it follows that $f^{\infty}$ is Lipschitz continuous in the last variable, uniformly with respect to $(x, s) \in \Omega \times \mathbb{R}^{N}$. 


\subsection{Setting of the problem and preliminary results}

For every $A \in \mathcal{A}(\Omega)$ and every $u \in \operatorname{BV}(\Omega)$, we set

$$
F(u, A)= \begin{cases}\int_{A} f(x, u, \nabla u) \mathrm{d} x & \text { if } u \in W^{1,1}(\Omega) \\ +\infty & \text { if } u \in \mathrm{BV}(\Omega) \backslash W^{1,1}(\Omega) .\end{cases}
$$

Our aim is to prove an integral representation theorem for the relaxation $\bar{F}$ of $F$, with respect to the $L^{1}$-topology, which is defined as the the greatest $L^{1}$-lower semicontinuous functional less than or equal to $F$. Namely, for every $A \in \mathcal{A}(\Omega)$,

$$
\bar{F}(u, A):=\inf \left\{\liminf _{n \rightarrow+\infty} F\left(u_{n}, A\right): u_{n} \in W^{1,1}(\Omega), u_{n} \rightarrow u \text { in } L^{1}(\Omega)\right\}
$$

For the properties of the relaxation we refer to $[8,12,16,17]$.

For every $A \in \mathcal{A}(\Omega)$, let us define the functional $\mathcal{F}_{f}(\cdot, A): \operatorname{BV}(\Omega) \rightarrow[0,+\infty)$ by setting

$$
\mathcal{F}_{f}(u, A)=\int_{A} f(x, u, \nabla u) \mathrm{d} x+\int_{A} f^{\infty}\left(x, \widetilde{u}, \frac{D^{c} u}{\left|D^{c} u\right|}\right) \mathrm{d}\left|D^{c} u\right|+\int_{J_{u} \cap A} \mathrm{~d} \mathcal{H}^{N-1} \int_{u^{-}(x)}^{u^{+}(x)} f^{\infty}\left(x, s, \nu_{u}\right) \mathrm{d} s .
$$

We shall often drop the subscript $f$ (if no confusion arises) and write $\mathcal{F}(u)$ in place of $\mathcal{F}_{f}(u, \Omega$ ).

The following two lower semicontinuity results will be useful in the sequel. The first one is an appropriate version of Theorem 1.1 in [14].

Theorem 2.9. Let $f: \Omega \times \mathbb{R} \times \mathbb{R}^{N} \rightarrow[0,+\infty)$ be a locally bounded Borel function, continuous with respect to $s$ and convex in $\xi$, satisfying the following assumptions:

$$
f(\cdot, s, \xi) \in W^{1,1}(\Omega) \quad \text { for all }(s, \xi) \in \mathbb{R} \times \mathbb{R}^{N}
$$

there exists a Borel set $N_{0} \subset \Omega$, with $\mathcal{H}^{N-1}\left(N_{0}\right)=0$, such that

$$
f(\cdot, s, \xi) \text { is approximately continuous in } \Omega \backslash N_{0} \text { for all }(s, \xi) \in \mathbb{R} \times \mathbb{R}^{N} \text {; }
$$

for every bounded set $B \subset \mathbb{R} \times \mathbb{R}^{N}$ there exists $L(B)>0$ such that

$$
\int_{\Omega}\left|\nabla_{x} f(x, s, \xi)\right| \mathrm{d} x<L(B) \quad \text { for all }(s, \xi) \in B
$$

Then, the functional $\mathcal{F}_{f}(\cdot, \Omega)$ defined in $(2.11)$ is $L^{1}$-lower semicontinuous in $\mathrm{BV}(\Omega)$.

Remark 2.10. Notice that assumption (2.12) may seem redundant, since every $W^{1,1}$-function admits a $\mathcal{H}^{N-1}$-a.e. approximately continuous representative. Moreover, the functional in (2.10) is clearly not affected by the choice of the representative. However, the functional (2.11) does depend on the particular representative chosen and it could be not lower semicontinuous for a different choice of it.

Notice that if $f, g_{k}: \mathbb{R}^{N} \rightarrow[0,+\infty)$ are convex functions such that $f=\sup _{k} g_{k}$, then from (2.6) we easily get that $f^{\infty}(\xi)=\sup _{k} g_{k}^{\infty}(\xi)$ for all $\xi$. This observation, combined with the argument used in the proof of Theorem 1.1 in [22] yields easily next result, which states that if $f$ is the supremum of a sequence of nonnegative integrands $g_{k}$ such that $\mathcal{F}_{g_{k}}$ is $L^{1}$-lower semicontinuous, then also $\mathcal{F}_{f}$ is lower semicontinuous. 
Lemma 2.11. Let $f, g_{k}: \Omega \times \mathbb{R} \times \mathbb{R}^{N} \rightarrow[0,+\infty), k \in \mathbb{N}$, be Borel functions, convex in the last variable and such that

$$
f(x, s, \xi)=\sup _{k \in \mathbb{N}} g_{k}(x, s, \xi) \quad \text { for all }(x, s, \xi) \in\left(\Omega \backslash N_{0}\right) \times \mathbb{R} \times \mathbb{R}^{N},
$$

where $N_{0} \subset \Omega$ is a Borel set with $\mathcal{H}^{N-1}\left(N_{0}\right)=0$. If the functionals $\mathcal{F}_{g_{k}}(\cdot, \Omega)$ are $L^{1}$-lower semicontinuous in $\mathrm{BV}(\Omega)$, then $\mathcal{F}_{f}(\cdot, \Omega)$ is $L^{1}$-lower semicontinuous in $\mathrm{BV}(\Omega)$, too.

\section{The LOWER SEMICONTINUITY RESUlts}

In this section we prove two lower semicontinuity theorems under very weak differentiability assumptions on $f$ with respect to the spatial variable $x$. To this aim, we need some approximation result of such an integrand with more regular functions.

First, we consider the special case of integrands which are split as a product of a BV function in $x$ times a function depending on $(s, \xi)$. In this case, the lower semicontinuity result is a simple consequence of the approximation Lemma 2.7. For the general case, we need to establish a suitable approximation result (see Lem. 3.3 below), which is very much in the spirit of the approximation result proved in [23].

\subsection{The case of separated variables}

Next result is immediately obtained from Lemma 2.7 and Theorem 2.9, via Lemma 2.11.

Theorem 3.1. Let $a: \Omega \rightarrow[0,+\infty)$ be a locally bounded $C_{1}$-quasi lower semicontinuous function. Let $p$ : $\mathbb{R} \times \mathbb{R}^{N} \rightarrow[0,+\infty)$ be a continuous function, convex with respect to the last variable. Then, the functional

$$
\mathcal{F}(u)=\int_{\Omega} a(x) p(u, \nabla u) \mathrm{d} x+\int_{\Omega} a(x) p^{\infty}\left(\widetilde{u}, \frac{D^{c} u}{D^{c} u \mid}\right)\left|D^{c} u\right|+\int_{J_{u} \cap \Omega} a(x) \mathrm{d} \mathcal{H}^{N-1} \int_{u^{-}(x)}^{u^{+}(x)} p^{\infty}\left(s, \nu_{u}\right) \mathrm{d} s
$$

is $L^{1}$-lower semicontinuous on $\mathrm{BV}(\Omega)$.

\subsection{The general case}

We start with a variant of Lemma 9.2 of [19] which can be proved with exactly the same argument. Therefore, we omit its simple proof.

Lemma 3.2. Let $X$ be a $\sigma$-compact metric space and $\mathcal{G}$ a family of lower semicontinuous functions $g: X \rightarrow \mathbb{R}$. Then, there exist a finite or countable subset $\mathcal{G}^{\prime} \subset \mathcal{G}$ such that

$$
\sup _{g \in \mathcal{G}^{\prime}} g(x)=\sup _{g \in \mathcal{G}} g(x) \quad \text { for all } x \in X .
$$

Next result is essentially contained in [23], Lemma 8 (c). However, we give here the proof for the sake of completeness.

Lemma 3.3. Let $X$ be a $\sigma$-compact subset of $\mathbb{R}^{d}$ and $f: X \times \mathbb{R}^{N} \rightarrow[0,+\infty)$ a function such that $f(z, \cdot)$ is convex for all $z \in X$. Let us assume also that for all $z_{0} \in X$ and $\varepsilon>0$, there exists $\delta>0$ such that

$$
f\left(z_{0}, \xi\right) \leq f(z, \xi)+\varepsilon(1+f(z, \xi)) \quad \text { for all }(z, \xi) \in X \times \mathbb{R}^{N} \text { such that }\left|z-z_{0}\right|<\delta .
$$

Then, there exist $\left\{a_{k}\right\} \subset C_{0}^{\infty}\left(\mathbb{R}^{d}\right)$ and $\left\{\psi_{k}\right\} \subset C^{\infty}\left(\mathbb{R}^{N}\right)$ such that, for all $k \in \mathbb{N}, 0 \leq a_{k} \leq 1, \psi_{k}$ is a convex function satisfying

$$
0 \leq \psi_{k}(\xi) \leq \Lambda_{k}(1+|\xi|) \quad \text { for all } \xi \in \mathbb{R}^{N}
$$


for some $\Lambda_{k}>0$, and

$$
f(z, \xi)=\sup _{k \in \mathbb{N}} a_{k}(z) \psi_{k}(\xi) \quad \text { for all }(z, \xi) \in X \times \mathbb{R}^{N}
$$

Proof. We start by noticing that if $X=\mathbb{R}^{d}$ and $f$, in addition to our assumptions, is also lower semicontinuous in $(z, \xi)$, then the assertion follows from [23], Lemma 8 (c). Thus, our task is to show that we may always reduce to this case.

To this aim, let us set $h=f+1$ and notice that from (3.1) it follows easily that

$$
\begin{aligned}
& \text { for all } z_{0} \in X \text { and } \varepsilon>0 \text { there exists } \delta>0 \text { such that } \\
& (1-\varepsilon) h\left(z_{0}, \xi\right) \leq h(z, \xi) \quad \text { for all }(z, \xi) \in X \times \mathbb{R}^{N} \text { such that }\left|z-z_{0}\right|<\delta \text {. }
\end{aligned}
$$

Then, we argue as in the proof of Proposition 9.3 in [19]. Let us denote by $\mathcal{G}$ the family of all continuous functions $g: \mathbb{R}^{d} \times \mathbb{R}^{N} \rightarrow[0,+\infty)$, convex with respect to the last variable, and satisfying the following conditions:

(i) $g(z, \xi) \leq h(z, \xi)$ for all $(z, \xi) \in X \times \mathbb{R}^{N}$;

(ii) $g$ satisfies $(3.3)$ in $\mathbb{R}^{d} \times \mathbb{R}^{N}$;

(iii) $0 \leq g(z, \xi) \leq \Lambda(1+|\xi|)$ for all $(z, \xi) \in \mathbb{R}^{d} \times \mathbb{R}^{N}$, for some $\Lambda>0$.

Let us now fix $z_{0} \in X, \varepsilon>0$, and let $\delta>0$ be such that (3.3) holds. Then, we choose $a \in C_{0}^{1}\left(B_{\delta}\left(z_{0}\right)\right)$ such that $0 \leq a \leq 1, a\left(z_{0}\right)=1$, and take a sequence of convex functions $\psi_{j}: \mathbb{R}^{n} \rightarrow[0,+\infty)$ satisfying (3.2) and such that $\sup _{j} \psi_{j}=h\left(z_{0}, \cdot\right)$. Clearly, the functions $g_{j}(z, \xi)=(1-\varepsilon) a(z) \psi_{j}(\xi)$ satisfy (i), (ii), (iii), and, by construction, $\sup _{j} g_{j}\left(z_{0}, \xi\right)=(1-\varepsilon) h\left(z_{0}, \xi\right)$ for all $\xi \in \mathbb{R}^{N}$. Therefore, we may conclude that

$$
\sup _{g \in \mathcal{G}} g(z, \xi) \geq h(z, \xi) \quad \text { for all }(z, \xi) \in X \times \mathbb{R}^{N}
$$

Since the opposite inequality follows immediately from (i), we get that in (3.4) the equality holds. Thus, using Lemma 3.2 we may conclude that there exists a sequence $\left\{g_{n}\right\} \subset \mathcal{G}$ such that

$$
\sup _{n \in \mathbb{N}} g_{n}(z, \xi)=h(z, \xi) \quad \text { for all }(z, \xi) \in X \times \mathbb{R}^{N}
$$

Setting now $f_{n}=\max \left\{g_{n}-1,0\right\}$, the functions $f_{n}$ are continuous in $\mathbb{R}^{d} \times \mathbb{R}^{N}$, convex in the last variable and satisfy (iii) for suitable positive constants $\Lambda_{n}$. Moreover it can be easily checked that each function $f_{n}$ satisfy (3.1) in $\mathbb{R}^{d} \times \mathbb{R}^{N}$ and, by construction,

$$
\sup _{n \in \mathbb{N}} f_{n}(z, \xi)=f(z, \xi) \quad \text { for all }(z, \xi) \in X \times \mathbb{R}^{N}
$$

The assertion then follows immediately by applying the approximation result stated in Lemma 8 (c) in [23] to each function $f_{n}$.

Next theorem is the main $L^{1}$-lower semicontinuity result of the paper. It is established without any hypothesis of coercivity or continuity with respect to $x$. More precisely, we assume only a $C_{1}$-quasi lower semicontinuity with respect to the spatial variable, with a suitable uniformity condition (see (3.5) below). On the other hand, we have to impose the growth assumption (2.8) which may appear not natural in the context of the lower semicontinuity. Notice however that this is not a big issue in view of the application of Theorem 3.4 to BV-relaxation.

Theorem 3.4. Let $f: \Omega \times \mathbb{R} \times \mathbb{R}^{N} \rightarrow[0,+\infty)$ be a Borel function such that $f(x, s, \cdot)$ is convex for every $(x, s) \in \Omega \times \mathbb{R}$ and $f(x, \cdot, \xi)$ is continuous for every $(x, \xi) \in \Omega \times \mathbb{R}^{N}$, satisfying (2.8). Moreover, assume that 
for any $h \in \mathbb{N}$ there exists an open set $A_{h} \subset \Omega$, with $C_{1}\left(A_{h}\right)<1 / h$, such that for every $\left(x_{0}, s_{0}\right) \in\left(\Omega \backslash A_{h}\right) \times \mathbb{R}$ and every $\varepsilon>0$, there exists $\delta>0$ such that

$$
f\left(x_{0}, s_{0}, \xi\right) \leq f(x, s, \xi)+\varepsilon(1+f(x, s, \xi))
$$

for all $(x, s, \xi) \in\left(\Omega \backslash A_{h}\right) \times \mathbb{R} \times \mathbb{R}^{N}$ such that $\left|x-x_{0}\right|+\left|s-s_{0}\right|<\delta$. Then, the functional $\mathcal{F}: \operatorname{BV}(\Omega) \rightarrow[0,+\infty)$ defined in (2.11) is $L^{1}$-lower semicontinuous on $\mathrm{BV}(\Omega)$.

Proof. Without loss of generality, we may assume that the sequence $\left\{A_{h}\right\}$ is such that $A_{h+1} \subset A_{h}$ for all $h \in \mathbb{N}$. Therefore, setting $A=\cap_{h} A_{h}$, we have $C_{1}(A)=\mathcal{H}^{N-1}(A)=0$.

For any $h$, recalling assumptions (3.5) and (2.8), we may apply Lemma 3.3 to the function $f$, with $X=\Omega \backslash A_{h}$, thus getting for any $h$ a sequence of functions $a_{k}^{h} \in C_{0}^{\infty}\left(\mathbb{R}^{N} \times \mathbb{R}\right)$, with $0 \leq a_{k}^{h} \leq 1$, and a sequence of convex functions $\psi_{k}^{h} \in C^{\infty}\left(\mathbb{R}^{N}\right)$ satisfying for all $k \in \mathbb{N}$

$$
0 \leq \psi_{k}^{h}(\xi) \leq \Lambda(1+|\xi|) \quad \text { for all } \xi \in \mathbb{R}^{N},
$$

where $\Lambda$ is the same constant appearing in (2.8), and such that

$$
f(x, s, \xi)=\sup _{k \in \mathbb{N}} a_{k}^{h}(x, s) \psi_{k}^{h}(\xi) \quad \text { for all }(x, s, \xi) \in\left(\Omega \backslash A_{h}\right) \times \mathbb{R} \times \mathbb{R}^{N} .
$$

Now, for every $h \in \mathbb{N}$, let $\varphi_{h} \in W^{1,1}\left(\mathbb{R}^{N}\right)$ be a capacitary quasi-potential of $A_{h}$. More precisely, let us assume that there exists a Borel set $N_{h} \subset \mathbb{R}^{N}$, with $C_{1}\left(N_{h}\right)=\mathcal{H}^{N-1}\left(N_{h}\right)=0$, such that $0 \leq \widetilde{\varphi}_{h}(x) \leq 1$ for every $x \in \mathbb{R}^{N} \backslash N_{h}, \widetilde{\varphi}_{h}=1$ on $A_{h} \backslash N_{h}$ and

$$
\int_{\mathbb{R}^{N}}\left|\nabla \widetilde{\varphi}_{h}\right| \mathrm{d} x \leq C_{1}\left(A_{h}\right)+\frac{1}{h}<\frac{2}{h}
$$

Let us now set, for all $h, k \in \mathbb{N}, \widetilde{\alpha}_{k}^{h}(x, s)=\max \left\{a_{k}^{h}(x, s)-\widetilde{\varphi}_{h}(x), 0\right\}$ for all $(x, s) \in\left(\Omega \backslash N_{h}\right) \times \mathbb{R}, \widetilde{\alpha}_{k}^{h}(x, s)=0$, otherwise. We have that

$$
0 \leq \widetilde{\alpha}_{k}^{h}(x, s) \leq 1, \quad a_{k}^{h}(x, s) \geq \widetilde{\alpha}_{k}^{h}(x, s) \geq a_{k}^{h}(x, s)-\widetilde{\varphi}_{h}(x) \quad \text { for all }(x, s) \in\left(\Omega \backslash N_{h}\right) \times \mathbb{R} .
$$

Moreover, setting $N_{0}=\cup_{h} N_{h}, C_{1}\left(N_{0}\right)=\mathcal{H}^{N-1}\left(N_{0}\right)=0$ and, for every $h, k \in \mathbb{N}$, we have that

$$
f(x, s, \xi) \geq \widetilde{\alpha}_{k}^{h}(x, s) \psi_{k}^{h}(\xi) \quad \text { for all }(x, s, \xi) \in\left(\Omega \backslash N_{0}\right) \times \mathbb{R} \times \mathbb{R}^{N} .
$$

In fact, if $x \in\left(\Omega \backslash A_{h}\right) \backslash N_{0}$, (3.9) follows from (3.7) and from the fact that $\widetilde{\varphi}_{h}(x) \geq 0$, while, if $x \in A_{h} \backslash N_{0}$, (3.9) holds since $\widetilde{\varphi}_{h}(x)=1$, hence $\widetilde{\alpha}_{k}^{h}(x, s)=0$. Finally, we set for all $h, k \in \mathbb{N}$

$$
g_{k}^{h}(x, s, \xi)=\widetilde{\alpha}_{k}^{h}(x, s) \psi_{k}^{h}(\xi), \quad g_{h}(x, s, \xi)=\sup _{k \in \mathbb{N}} g_{k}^{h}(x, s, \xi), \quad f_{h}(x, s, \xi)=\sup _{k \in \mathbb{N}} a_{k}^{h}(x, s) \psi_{k}^{h}(\xi)
$$

for all $(x, s, \xi) \in \Omega \times \mathbb{R} \times \mathbb{R}^{N}$. Notice that each function $g_{k}^{h}$ satisfies the assumptions of Theorem 2.9. Therefore, the functionals $\mathcal{F}_{g_{k}^{h}}(\cdot, \Omega)$ are all $L^{1}$-lower semicontinuous in $\mathrm{BV}(\Omega)$, hence by Lemma 2.11 the same is true for the functionals $\mathcal{F}_{g_{h}}(\cdot, \Omega)$, for any $h \in \mathbb{N}$.

To prove the lower semicontinuity of $\mathcal{F}_{f}$, let us take a sequence $\left\{u_{j}\right\} \subset \operatorname{BV}(\Omega)$ converging in $L^{1}(\Omega)$ to $u \in \mathrm{BV}(\Omega)$. Let us fix $h \in \mathbb{N}$ and set

$$
\psi_{h}(\xi)=\sup _{k \in \mathbb{N}} \psi_{k}^{h}(\xi) \quad \text { for all } \xi \in \mathbb{R}^{N}
$$


From (3.9), (3.8) and (3.7), we then get that

$$
\begin{aligned}
\liminf _{j \rightarrow+\infty} \mathcal{F}_{f}\left(u_{j}, \Omega\right) \geq & \liminf _{j \rightarrow+\infty} \mathcal{F}_{g_{h}}\left(u_{j}, \Omega\right) \geq \mathcal{F}_{g_{h}}(u, \Omega) \geq \mathcal{F}_{f_{h}}(u, \Omega)-\int_{\Omega} \widetilde{\varphi}_{h} \psi_{h}(\nabla u) \mathrm{d} x \\
& -\int_{\Omega} \widetilde{\varphi}_{h} \psi_{h}^{\infty}\left(\frac{D^{c} u}{\left|D^{c} u\right|}\right) \mathrm{d}\left|D^{c} u\right|-\int_{J_{u} \cap \Omega} \mathrm{d} \mathcal{H}^{N-1} \int_{u^{-}(x)}^{u^{+}(x)} \widetilde{\varphi}_{h} \psi_{h}^{\infty}(\nu) \mathrm{d} s \\
\geq & \mathcal{F}_{f}\left(u, \Omega \backslash A_{h}\right)-\int_{\Omega} \widetilde{\varphi}_{h} \psi_{h}(\nabla u) \mathrm{d} x-\int_{\Omega} \widetilde{\varphi}_{h} \psi_{h}^{\infty}\left(\frac{D^{s} u}{\left|D^{s} u\right|}\right) \mathrm{d}\left|D^{s} u\right| .
\end{aligned}
$$

Thus, recalling (3.6), we obtain

$$
\liminf _{j \rightarrow+\infty} \mathcal{F}_{f}\left(u_{j}, \Omega\right) \geq \mathcal{F}_{f}\left(u, \Omega \backslash A_{h}\right)-\Lambda \int_{\Omega} \widetilde{\varphi}_{h}(1+|\nabla u|) \mathrm{d} x-\Lambda \int_{\Omega} \widetilde{\varphi}_{h} \mathrm{~d}\left|D^{s} u\right| .
$$

Since $\widetilde{\varphi}_{h} \rightarrow 0$ in $W^{1,1}\left(\mathbb{R}^{N}\right)$ as $h \rightarrow \infty$, we have that, up to a subsequence, $\widetilde{\varphi}_{h}(x) \rightarrow 0$ for $\mathcal{H}^{N-1}$-almost every $x \in \mathbb{R}^{N}$ (see Prop. 1.2 in [11]). Therefore, letting $h \rightarrow+\infty$ in (3.10) and recalling that $A_{h+1} \subset A_{h}$ for all $h$ and that $\mathcal{H}^{N-1}\left(\cap_{h} A_{h}\right)=0$, from the dominated convergence theorem we get that

$$
\liminf _{j \rightarrow+\infty} \mathcal{F}_{f}\left(u_{j}, \Omega\right) \geq \mathcal{F}_{f}(u, \Omega)
$$

thus proving the assertion.

As an application of the previous theorem we are going to prove a fairly general lower semicontinuity result for an integrand $f$ depending only on $x$ and $\xi$. To this aim, let us recall the following approximation result proved in [24], Theorem 4.

Theorem 3.5. Let $\Omega$ be an open subset of $\mathbb{R}^{N}, f: \Omega \times \mathbb{R}^{N} \rightarrow[0,+\infty)$ a function such for every $x \in \Omega, f(x, \cdot)$ is convex and demicoercive. Let us set, for any $\xi_{0} \in \mathbb{R}^{N}$,

$$
\left(P_{\xi_{0}} f\right)(x, \xi)=-1+\inf _{\lambda>0}\left\{\lambda+\lambda f\left(x, \xi_{0}+\frac{\xi-\xi_{0}}{\lambda}\right)\right\} \quad \text { for all }(x, \xi) \in \Omega \times \mathbb{R}^{N} .
$$

Then, for every $x \in \Omega,\left(P_{\xi_{0}} f\right)(x, \cdot)$ is convex and demicoercive and $\xi \mapsto 1+\left(P_{\xi_{0}} f\right)\left(x, \xi+\xi_{0}\right)$ is a 1-positively homogeneous function. Moreover

$$
f(x, \xi)=\sup _{\xi_{0} \in \mathbb{R}^{N}}\left(P_{\xi_{0}} f\right)(x, \xi) \quad \text { for all }(x, \xi) \in \Omega \times \mathbb{R}^{N} .
$$

Furthermore, if $f$ is lower semicontinuous in $\Omega \times \mathbb{R}^{N}$, then $P_{\xi_{0}} f$ is lower semicontinuous, too.

Theorem 3.6. Let $f: \Omega \times \mathbb{R}^{N} \rightarrow[0,+\infty)$ be a locally bounded Borel function such that $f(x, \cdot)$ is convex for every $x \in \Omega$ and $f(\cdot, \xi)$ is $C_{1}$-quasi lower semicontinuous for every $\xi \in \mathbb{R}^{N}$. Moreover, assume that at least one of the following two conditions holds:

$$
\begin{aligned}
& \left\{\begin{array}{l}
f(x, \cdot) \text { is positively } 1 \text {-homogeneous and } \\
f(x, \xi)>0 \text { for all }(x, \xi) \in\left(\Omega \backslash N_{0}\right) \times\left(\mathbb{R}^{N} \backslash\{0\}\right), \text { where } \mathcal{H}^{N-1}\left(N_{0}\right)=0 ;
\end{array}\right. \\
& \left\{\begin{array}{l}
f \text { satisfies }(2.8) \text { and there exists } \Psi: \mathbb{R}^{N} \rightarrow[0,+\infty), \text { convex and demicoercive, } \\
\text { such that } \Psi(\xi) \leq f(x, \xi) \text { for all }(x, \xi) \in \Omega \times \mathbb{R}^{N}
\end{array}\right.
\end{aligned}
$$

Then, the functional $\mathcal{F}_{f}$ is $L^{1}$-lower semicontinuous on $\mathrm{BV}(\Omega)$. 
Proof.

Step 1. Let us prove the assertion under the assumption (3.13). To this aim, notice that since $f$ is locally bounded in $\Omega \times \mathbb{R}^{N}$ and positively 1-homogeneous with respect to $\xi$, for any open set $\Omega^{\prime} \subset \subset \Omega$, there exists a constant $\Lambda^{\prime}$ such that

$$
0 \leq f(x, \xi) \leq \Lambda^{\prime}|\xi| \quad \text { for all }(x, \xi) \in \Omega^{\prime} \times \mathbb{R}^{N}
$$

This estimate, together with the convexity of $f$ with respect to $\xi$ immediately yields that

$$
\left|f\left(x, \xi_{1}\right)-f\left(x, \xi_{2}\right)\right| \leq c \Lambda^{\prime}\left|\xi_{1}-\xi_{2}\right| \quad \text { for all }\left(x, \xi_{1}\right),\left(x, \xi_{2}\right) \in \Omega^{\prime} \times \mathbb{R}^{N},
$$

for some constant $c$ depending only on the dimension $N$. Let us now fix $h$ and a sequence $\left\{\xi_{j}\right\}$ dense in $\mathbb{R}^{N}$. For all $j$ there exists an open set $A_{j, h} \subset \Omega, A_{j, h} \supset N_{0}$, with $C_{1}\left(A_{j, h}\right)<1 /\left(h 2^{j}\right)$, such that $f\left(\cdot, \xi_{j}\right)$ is lower semicontinuous in $\Omega \backslash A_{j, h}$. Setting $A_{h}=\cup_{j} A_{j, h}, A_{h}$ is open, $C_{1}\left(A_{h}\right)<1 / h$, and making use of (3.15), one easily gets that $f$ is lower semicontinuous in $\left(\Omega^{\prime} \backslash A_{h}\right) \times \mathbb{R}^{N}$.

In order to prove that the functional $\mathcal{F}_{f}\left(\cdot, \Omega^{\prime}\right)$ is $L^{1}$-lower semicontinuous in $\mathrm{BV}\left(\Omega^{\prime}\right)$, by Theorem 3.4 it is enough to show that, given $h$ and $x_{0} \in \Omega^{\prime} \backslash A_{h}$, for all $\varepsilon>0$ there exists $\delta>0$ such that

$$
f\left(x_{0}, \xi\right) \leq(1+\varepsilon) f(x, \xi) \quad \text { for all }(x, \xi) \in\left(\Omega^{\prime} \backslash A_{h}\right) \times \mathbb{R}^{N} \text { such that }\left|x-x_{0}\right|<\delta .
$$

To prove this, we argue by contradiction, assuming that there exist $x_{0} \in \Omega^{\prime} \backslash A_{h}$ and $\varepsilon_{0}>0$ such that for any $k \in \mathbb{N}$, there exist two sequences $x_{k} \in \Omega^{\prime} \backslash A_{h}$, with $\left|x_{k}-x_{0}\right|<1 / k$, and $\xi_{k} \in \mathbb{R}^{N}$ such that

$$
f\left(x_{0}, \xi_{k}\right)>\left(1+\varepsilon_{0}\right) f\left(x_{k}, \xi_{k}\right)
$$

Clearly, by the positive 1-homogeneity of $f(x, \cdot)$, we may assume that $\left|\xi_{k}\right|=1$, for every $k \in \mathbb{N}$; hence, up to a subsequence, there exists $\xi_{0} \in \mathbb{S}^{N-1}$ such that $\xi_{k} \rightarrow \xi_{0}$. Then, passing to the limit when $k \rightarrow+\infty$ in $(3.16)$ and using the lower semicontinuity of $f$ and the continuity of $f\left(x_{0}, \cdot\right)$, we get that

$$
f\left(x_{0}, \xi_{0}\right)=\lim _{k \rightarrow+\infty} f\left(x_{0}, \xi_{k}\right) \geq\left(1+\varepsilon_{0}\right) \liminf _{k \rightarrow+\infty} f\left(x_{k}, \xi_{k}\right) \geq\left(1+\varepsilon_{0}\right) f\left(x_{0}, \xi_{0}\right) .
$$

Hence, $f\left(x_{0}, \xi_{0}\right)=0$, which is a contradiction since $x_{0} \in \Omega \backslash N_{0}$.

This proves that $\mathcal{F}_{f}\left(\cdot, \Omega^{\prime}\right)$ is lower semicontinuous and, by letting $\Omega^{\prime} \uparrow \Omega$, the lower semicontinuity of $\mathcal{F}_{f}(\cdot, \Omega)$ follows.

Step 2. Assume that (3.14) holds. Since (2.8) is in force, arguing as in the previous step, we have that for all $h \in \mathbb{N}$, there exists an open set $A_{h} \subset \Omega$, with $C_{1}\left(A_{h}\right)<1 / h$, such that $f$ is lower semicontinuous in $\left(\Omega \backslash A_{h}\right) \times \mathbb{R}^{N}$.

For all $\xi_{0} \in \mathbb{R}^{N}$, let us consider the function $P_{\xi_{0}} f$ defined in (3.11). Since $f$ is lower semicontinuous in $\left(\Omega \backslash A_{h}\right) \times \mathbb{R}^{N}$, from the last assertion of Theorem 3.5 (which actually holds also if $\Omega$ is replaced by any $\sigma$ compact subset of $\mathbb{R}^{N}$ ) we have that $P_{\xi_{0}} f$ is lower semicontinuous in $\left(\Omega \backslash A_{h}\right) \times \mathbb{R}^{N}$ for any $h$. Therefore, recalling (3.12) and using Lemma 3.2, we get that for any $h$ there exists a sequence $\left\{\xi_{k}^{h}\right\}_{k \in \mathbb{N}} \subset \mathbb{R}^{N}$ such that

$$
f(x, \xi)=\sup _{k \in \mathbb{N}}\left(P_{\xi_{k}^{h}} f\right)(x, \xi) \quad \text { for all }(x, \xi) \in\left(\Omega \backslash A_{h}\right) \times \mathbb{R}^{N} .
$$

Thus, by relabelling the sequence $\left\{\xi_{k}^{h}\right\}_{(h, k) \in \mathbb{N} \times \mathbb{N}}$, we may conclude that there exists a sequence $\left\{\xi_{n}\right\}_{n \in \mathbb{N}}$ such that

$$
f(x, \xi)=\sup _{n \in \mathbb{N}}\left(P_{\xi_{n}} f\right)(x, \xi) \quad \text { for all }(x, \xi) \in\left(\Omega \backslash A_{0}\right) \times \mathbb{R}^{N},
$$

where $A_{0}=\cap_{h} A_{h}$. Let us set, for all $n$,

$$
\Psi_{n}(\xi)=\left(P_{\xi_{n}} \Psi\right)\left(\xi+\xi_{n}\right)+1 \quad \text { for all } \xi \in \mathbb{R}^{N}
$$


Since, by Theorem 3.5 the functions $\Psi_{n}$ are all demicoercive and positively 1-homogeneous, for every $n$ there exist $a_{n}>0$ and $v_{n} \in \mathbb{R}^{N}$ such that

$$
a_{n}|\xi| \leq \Psi_{n}(\xi)+\left\langle v_{n}, \xi\right\rangle \quad \text { for all } \xi \in \mathbb{R}^{N} .
$$

Therefore, setting $g_{n}(x, \xi)=\left(P_{\xi_{n}} f\right)\left(x, \xi+\xi_{n}\right)+1$, we may conclude that

$$
a_{n}|\xi| \leq g_{n}(x, \xi)+\left\langle v_{n}, \xi\right\rangle \quad \text { for all }(x, \xi) \in \Omega \times \mathbb{R}^{N} .
$$

The functions $\widehat{g}_{n}(x, \xi)=g_{n}(x, \xi)+\left\langle v_{n}, \xi\right\rangle$ are $C_{1}$-quasi lower semicontinuous in $x$ and positively homogeneous in $\xi$ and from (3.18) it is clear that they all satisfy (3.13). Therefore, from what we have proved in Step 1 we may conclude that the functionals $\mathcal{F}_{\widehat{g}_{n}}(\cdot, \Omega)$ are $L^{1}$-lower semicontinuous in $\mathrm{BV}(\Omega)$.

Let us now prove that also the functionals $\mathcal{F}_{g_{n}}(\cdot, \Omega)$ are lower semicontinuous. To this aim, let us take a sequence of functions $u_{j} \in \mathrm{BV}(\Omega)$ converging in $L^{1}(\Omega)$ to $u \in \mathrm{BV}(\Omega)$. Let us fix an open set $\Omega^{\prime} \subset \subset \Omega$ and a function $\psi \in C_{0}^{1}(\Omega), 0 \leq \psi \leq 1$, such that $\psi \equiv 1$ in $\Omega^{\prime}$. We have, using the fact that $g_{n}, \psi \geq 0, \psi \equiv 1$ in $\Omega^{\prime}$, and integrating by parts twice,

$$
\begin{aligned}
\liminf _{j \rightarrow+\infty} \mathcal{F}_{g_{n}}\left(u_{j}, \Omega\right) & =\liminf _{j \rightarrow+\infty}\left[\mathcal{F}_{g_{n}}\left(u_{j}, \Omega\right)+\int_{\Omega} \psi\left\langle v_{n}, D u_{j}\right\rangle-\int_{\Omega} \psi\left\langle v_{n}, D u_{j}\right\rangle\right] \\
& \geq \liminf _{j \rightarrow+\infty}\left[\int_{\Omega} \psi g_{n}\left(x, \frac{D u_{j}}{\left|D u_{j}\right|}\right)\left|D u_{j}\right|+\int_{\Omega} \psi\left\langle v_{n}, D u_{j}\right\rangle-\int_{\Omega} \psi\left\langle v_{n}, D u_{j}\right\rangle\right] \\
& \geq \liminf _{j \rightarrow+\infty} \mathcal{F}_{\widehat{g}_{n}}\left(u_{j}, \Omega^{\prime}\right)+\lim _{j \rightarrow+\infty} \int_{\Omega} u_{j}\left\langle v_{n}, \nabla \psi\right\rangle \mathrm{d} x \\
& \geq \mathcal{F}_{\widehat{g}_{n}}\left(u, \Omega^{\prime}\right)+\int_{\Omega} u\left\langle v_{n}, \nabla \psi\right\rangle \mathrm{d} x=\mathcal{F}_{\widehat{g}_{n}}\left(u, \Omega^{\prime}\right)-\int_{\Omega} \psi\left\langle v_{n}, D u\right\rangle \\
& =\mathcal{F}_{g_{n}}\left(u, \Omega^{\prime}\right)-\int_{\Omega \backslash \Omega^{\prime}} \psi\left\langle v_{n}, D u\right\rangle .
\end{aligned}
$$

The lower semicontinuity of $\mathcal{F}_{g_{n}}(\cdot, \Omega)$ follows by letting first $\psi \uparrow 1$ and then $\Omega^{\prime} \uparrow \Omega$.

Set now $h_{n}(x, \xi)=g_{n}\left(x, \xi-\xi_{n}\right)$ and observe that $h_{n}^{\infty}(x, \xi)=g_{n}(x, \xi)$ for all $(x, \xi) \in \Omega \times \mathbb{R}^{N}$. Thus,

$$
\mathcal{F}_{h_{n}}(u, \Omega)=\mathcal{F}_{g_{n}}\left(u-\left\langle\xi_{n}, \cdot\right\rangle, \Omega\right) \quad \text { for all } u \in \operatorname{BV}(\Omega),
$$

hence $\mathcal{F}_{h_{n}}(\cdot, \Omega)$ is lower semicontinuous, too. This fact, thanks to (3.17) and to Lemma 2.11 immediately implies the $L^{1}$-lower semicontinuity of $\mathcal{F}_{f+1}(\cdot, \Omega)$, hence the assertion follows.

\section{Relaxation}

This section is devoted to relaxation results.

The first one (Th. 4.1) concerns the case of separated variables, under the assumption of $C_{1}$-quasi continuity of the integrand with respect to $x$. This theorem improves the relaxation result obtained, under stronger regularity assumptions, in [2]. Its proof is based on a Reshetnyak-type result which is a consequence of the lower semicontinuity Theorem 3.1, and follows the same outlines as in [2].

On the other hand, in Sections 4.2 and 4.3 we consider the general case and prove in particular Theorem 1.1, which is the main relaxation result of this paper. In that theorem we state the classical relaxation formula (1.7), under the very weak assumption of BV dependence of the integrand with respect to $x$. As usual, this result is attained, once we have proved the so-called "liminf" and "limsup" inequalities. Since the first one is a consequence of the lower semicontinuity results contained in Section 3.2, it is sufficient to prove here only the "lim sup" inequality (Th. 4.2). In order to achieve this result, we adapt the blow-up technique introduced by Fonseca-Muller in [20] and [21] in the case of continuous integrands; however, in our case, this is a very delicate 
technical point. Indeed, the presence of jump discontinuities of the integrand, due to the assumption of BV dependence in $x$, imposes a refined use of the trace properties of $\mathrm{BV}$ functions.

\subsection{The case of separated variables under $C_{1}$-quasi continuity assumption}

Theorem 4.1. Let $a: \Omega \rightarrow[0,+\infty)$ be a bounded $C_{1}$-quasi continuous function and let $p: \mathbb{R} \times \mathbb{R}^{N} \rightarrow[0,+\infty)$ be a continuous function, convex in the last variable and such that

$$
0 \leq p(s, \xi) \leq \Lambda(1+|\xi|) \quad \text { for every }(s, \xi) \in \mathbb{R} \times \mathbb{R}^{N},
$$

for some $\Lambda>0$. Let $F: \operatorname{BV}(\Omega) \rightarrow[0,+\infty]$ be the functional defined by

$$
F(u)= \begin{cases}\int_{\Omega} a(x) p(u, \nabla u) \mathrm{d} x & \text { if } u \in W^{1,1}(\Omega) \\ +\infty & \text { if } u \in \mathrm{BV}(\Omega) \backslash W^{1,1}(\Omega),\end{cases}
$$

and $\bar{F}$ be the relaxation of $F$. Then, for every $u \in \operatorname{BV}(\Omega)$,

$$
\bar{F}(u)=\int_{\Omega} a(x) p(u, \nabla u) \mathrm{d} x+\int_{\Omega} a(x) p^{\infty}\left(\widetilde{u}, \frac{D^{c} u}{\left|D^{c} u\right|}\right)\left|D^{c} u\right|+\int_{J_{u} \cap \Omega} a(x) \mathrm{d} \mathcal{H}^{N-1} \int_{u^{-}(x)}^{u^{+}(x)} p^{\infty}\left(s, \nu_{u}\right) \mathrm{d} s .
$$

Proof. Let us denote by $\mathcal{F}$ the functional on the right hand side of (4.3). Clearly, $\mathcal{F} \leq F$ and, by Theorem 3.1, it follows that $\mathcal{F}$ is $L^{1}$-lower semicontinuous on $\operatorname{BV}(\Omega)$, so that $\mathcal{F} \leq \bar{F}$. Now we prove the opposite inequality.

Indeed, let $u \in \operatorname{BV}(\Omega)$ and $u_{h}=u * \phi_{h}$, where $\left\{\phi_{h}\right\}$ is a sequence of mollifiers. Then $\left\{u_{h}\right\} \subset W_{\text {loc }}^{1,1}(\Omega)$. Moreover, $u_{h} \rightarrow u$ strongly in $L_{\text {loc }}^{1}(\Omega)$ and $\int_{A}\left|\nabla u_{h}\right| \mathrm{d} x \rightarrow|D u|(A)$, for every $A \subset \subset \Omega$, such that $|D u|(A)=0$. Denoting by $\mathcal{G}\left(u_{h}\right)$ and $\mathcal{G}(u)$ the subgraphs of $u_{h}$ and $u$, respectively, by Theorems 1.8 and 1.10 in [27] and Proposition 1.1 in [9] we have that

$$
\chi_{\mathcal{G}\left(u_{h}\right)} \rightarrow \chi_{\mathcal{G}(u)} \quad \text { in } L_{\text {loc }}^{1}(\Omega \times \mathbb{R}) \quad \text { and } \quad\left|\alpha\left(u_{h}\right)\right|(A \times \mathbb{R}) \rightarrow|\alpha(u)|(A \times \mathbb{R}),
$$

where $\alpha\left(u_{h}\right)=D \chi_{\mathcal{G}\left(u_{h}\right)}$ and $\alpha(u)=D \chi_{\mathcal{G}(u)}$. Setting

$$
\widehat{p}(s, \xi, \tau):= \begin{cases}-p(s,-\xi / \tau) \tau & \text { if } \tau<0, \\ p^{\infty}(s, \xi) & \text { if } \tau=0,\end{cases}
$$

by Lemma 2.2 in [10], it follows that

$$
\lim _{h \rightarrow \infty} F\left(u_{h}, A\right)=\lim _{h \rightarrow \infty} \mathcal{F}\left(u_{h}, A\right)=\lim _{h \rightarrow \infty} \int_{A \times \mathbb{R}} a(x) \widehat{p}\left(s, \frac{\alpha\left(u_{h}\right)}{\left|\alpha\left(u_{h}\right)\right|}(x, s)\right) \mathrm{d}\left|\alpha\left(u_{h}\right)\right|(x, s) .
$$

Notice that by the classical Reshetnyak Theorem (see [28] or [4], Th. 2.39) we get

$$
\lim _{h \rightarrow \infty} \int_{A \times \mathbb{R}} \widehat{p}\left(s, \frac{\alpha\left(u_{h}\right)}{\left|\alpha\left(u_{h}\right)\right|}(x, s)\right) \mathrm{d}\left|\alpha\left(u_{h}\right)\right|(x, s)=\int_{A \times \mathbb{R}} \widehat{p}\left(s, \frac{\alpha(u)}{|\alpha(u)|}(x, s)\right) \mathrm{d}|\alpha(u)|(x, s) .
$$

Moreover by Theorem 3.1 we have

$$
\liminf _{h \rightarrow \infty} \int_{A \times \mathbb{R}} \widetilde{B}(x) \widehat{p}\left(s, \frac{\alpha\left(u_{h}\right)}{\left|\alpha\left(u_{h}\right)\right|}\right) \mathrm{d}\left|\alpha\left(u_{h}\right)\right| \geq \int_{A \times \mathbb{R}} \widetilde{B}(x) \widehat{p}\left(s, \frac{\alpha(u)}{|\alpha(u)|}\right) \mathrm{d}|\alpha(u)|,
$$


where $\widetilde{B}(x)$ can be chosen either equal to $a(x)$ or to $\lambda-a(x)$ with $\lambda=\operatorname{ess} \sup a(x)$. As a consequence of (4.5) and (4.6) we have

$$
\lim _{h \rightarrow \infty} \int_{A \times \mathbb{R}} a(x) \widehat{p}\left(s, \frac{\alpha\left(u_{h}\right)}{\left|\alpha\left(u_{h}\right)\right|}\right) \mathrm{d}\left|\alpha\left(u_{h}\right)\right|=\int_{A \times \mathbb{R}} a(x) \widehat{p}\left(s, \frac{\alpha(u)}{|\alpha(u)|}\right) \mathrm{d}|\alpha(u)|=\mathcal{F}(u, A) .
$$

Hence, from (4.7) and (4.4), it follows

$$
\bar{F}(u, A) \leq \liminf _{h \rightarrow+\infty} F\left(u_{h}, A\right)=\mathcal{F}(u, A) \leq \mathcal{F}(u) .
$$

By approximation we then have $\bar{F}(u) \leq \mathcal{F}(u)$ and the theorem is proven.

\subsection{The "lim sup" inequality in the general case}

In this section we will assume that the integrand $f$ does not depend on $s$.

Moreover, we will assume that the recession function $f^{\infty}: \Omega \times \mathbb{R}^{N} \rightarrow[0,+\infty)$ satisfies

$$
f^{\infty}(\cdot, \xi) \in \mathrm{BV}(\Omega) \quad \text { for every } \xi \in \mathbb{R}^{N},
$$

and that for every $\xi \in \mathbb{R}^{N}$ there exists $N_{\xi} \subset \Omega$, with $\mathcal{H}^{N-1}\left(N_{\xi}\right)=0$, such that

$$
f^{\infty}(x, \xi)=\left(f^{\infty}\right)^{-}(x, \xi) \quad \text { for all } x \in \Omega \backslash N_{\xi} .
$$

Theorem 4.2. Assume that $f: \Omega \times \mathbb{R}^{N} \rightarrow \mathbb{R}$ is a Borel function satisfying (2.7) and (2.8). Let $F: \operatorname{BV}(\Omega) \times$ $\mathcal{A}(\Omega) \rightarrow[0,+\infty]$ be the functional defined in $(2.10)$ and $\bar{F}$ be the relaxation of $F$. Assume also that (4.8) and (4.9) hold. Then, $\bar{F}(u, \cdot)$ is the trace of a finite Radon measure on $\mathcal{A}(\Omega)$, and, for every $A \in \mathcal{A}(\Omega)$ and every $u \in \mathrm{BV}(\Omega)$,

$$
\bar{F}(u, A) \leq \int_{A} f(x, \nabla u) \mathrm{d} x+\int_{A} f^{\infty}\left(x, \frac{D^{c} u}{\left|D^{c} u\right|}\right) \mathrm{d}\left|D^{c} u\right|+\int_{J_{u} \cap A}\left[u^{+}(x)-u^{-}(x)\right] f^{\infty}\left(x, \nu_{u}(x)\right) \mathrm{d} \mathcal{H}^{N-1} .
$$

We start by observing that under the assumptions of Theorem 4.2 above it is well known that for any $u \in \operatorname{BV}(\Omega)$ the function $\bar{F}(u, \cdot)$ is the trace of a finite Radon measure on $\mathcal{A}(\Omega)$ and that for all $A \in \mathcal{A}(\Omega)$

$$
0 \leq \bar{F}(u, A) \leq \Lambda\left(\mathcal{L}^{N}(A)+|D u|(A)\right) .
$$

Following [19], Proof of Theorem 1.3, we fix $u \in \mathrm{BV}(\Omega)$ and consider the Radon-Nikodým derivatives of $\bar{F}(u, \cdot)$ with respect to the Lebesgue measure $\mathcal{L}^{N}$, to the total variation of the Cantor measure $\left|D^{c} u\right|$ and to the Hausdorff measure $\mathcal{H}^{N-1}\left\lfloor S_{u}\right.$, respectively. In order to obtain Theorem 4.2, we will prove that

(L) $\quad \frac{\mathrm{d} \bar{F}(u, \cdot)}{\mathrm{d} \mathcal{L}^{N}}\left(x_{0}\right) \leq f\left(x_{0}, \nabla u\left(x_{0}\right)\right) \quad$ for $\mathcal{L}^{N}$-almost every $x_{0} \in \Omega$,

(C) $\quad \frac{\mathrm{d} \bar{F}(u, \cdot)}{\mathrm{d}\left|D^{c} u\right|}\left(x_{0}\right) \leq f^{\infty}\left(x_{0}, \frac{D^{c} u}{\left|D^{c} u\right|}\left(x_{0}\right)\right) \quad$ for $\left|D^{c} u\right|$-almost every $x_{0} \in \Omega$,

(J) $\quad \frac{\mathrm{d} \bar{F}(u, \cdot)}{\mathrm{d} \mathcal{H}^{N-1}\left\lfloor J_{u}\right.}\left(x_{0}\right) \leq\left[u^{+}\left(x_{0}\right)-u^{-}\left(x_{0}\right)\right] f^{\infty}\left(x_{0}, \nu_{u}\left(x_{0}\right)\right) \quad$ for $\mathcal{H}^{N-1}$-almost every $x_{0} \in J_{u}$.

Inequality (L) is proven in [19], Theorem 1.3, part (i), under assumptions (2.7) and (2.8) alone, hence, we have to prove $(\mathrm{C})$ and $(\mathrm{J})$. To this purpose let us define the following coercive functional associated to $\bar{F}$ by setting

$$
\bar{F}_{1}(u, A):=\bar{F}(u, A)+|D u|(A) .
$$


Proposition 4.3. Let $f$ satisfy the assumptions of Theorem 4.2. Then, $(C)$ holds; i.e., for every $u \in \mathrm{BV}(\Omega)$,

$$
\frac{\mathrm{d} \bar{F}(u, \cdot)}{\mathrm{d}\left|D^{c} u\right|}\left(x_{0}\right) \leq f^{\infty}\left(x_{0}, \frac{D^{c} u}{\left|D^{c} u\right|}\left(x_{0}\right)\right) \quad \text { for }\left|D^{c} u\right| \text {-almost every } x_{0} \in \Omega .
$$

Proof. By Lemma 3.9 of [7] for $\left|D^{c} u\right|$-almost every $x_{0} \in \Omega$, there exists a double indexed sequence $\left\{t_{\varepsilon}^{h}, u_{\varepsilon}^{h}\right\}$ such that, for every $h \in \mathbb{N}$,

$$
t_{\varepsilon}^{h} \rightarrow+\infty, \quad \varepsilon t_{\varepsilon}^{h} \rightarrow 0^{+}, \quad u_{\varepsilon}^{h} \rightarrow \widetilde{u}\left(x_{0}\right) \quad \text { as } \varepsilon \rightarrow 0^{+},
$$

$$
\begin{aligned}
& \frac{\mathrm{d} \bar{F}_{1}(u, \cdot)}{\mathrm{d}\left|D^{c} u\right|}\left(x_{0}\right)=\frac{\mathrm{d} \bar{F}(u, \cdot)}{\mathrm{d}\left|D^{c} u\right|}\left(x_{0}\right)+1 \\
& \quad=\lim _{h \rightarrow+\infty} \limsup _{\varepsilon \rightarrow 0^{+}} \frac{\inf \left\{\bar{F}_{1}\left(v, Q_{\nu}^{h}\left(x_{0}, \varepsilon\right)\right): v \in \operatorname{BV}\left(Q_{\nu}^{h}\left(x_{0}, \varepsilon\right)\right),\left.v\right|_{\partial Q_{\nu}^{h}\left(x_{0}, \varepsilon\right)}=u_{\varepsilon}^{h}+\left\langle t_{\varepsilon}^{h} \nu, x-x_{0}\right\rangle\right\}}{h^{N-1} \varepsilon^{N} t_{\varepsilon}^{h}},
\end{aligned}
$$

where $\nu=\frac{D^{c} u}{\left|D^{c} u\right|}\left(x_{0}\right),|\nu|=1$, and $Q_{\nu}^{h}\left(x_{0}, \varepsilon\right):=x_{0}+\varepsilon Q_{\nu}^{h}$, with

$$
Q_{\nu}^{h}:=R_{\nu}\left((-h / 2, h / 2)^{N-1} \times(-1 / 2,1 / 2)\right),
$$

and $R_{\nu}$ denotes a rotation such that $R_{\nu} e_{N}=\nu$.

Let $N_{1} \subseteq \Omega \backslash S_{u}$, with $\left|D^{c} u\right|\left(N_{1}\right)=0$, be such that for every $x_{0} \in\left(\Omega \backslash S_{u}\right) \backslash N_{1}$ (4.10) holds and all the limits above exist and are finite.

Let $D_{0}=\left\{\nu_{j}\right\}$ be a countable dense subset of $\mathbb{S}^{N-1}$. Moreover, let $N_{2} \subseteq \Omega$ defined by

$$
N_{2}:=\bigcup_{j \in \mathbb{N}}\left[S_{f \infty\left(\cdot, \nu_{j}\right)} \cup N_{\nu_{j}}\right]
$$

where, for every $\nu_{j} \in D_{0}, S_{f^{\infty}\left(\cdot, \nu_{j}\right)}$ is the approximate singular set of the BV-function $f^{\infty}\left(\cdot, \nu_{j}\right)$ and $N_{\nu_{j}}$ is defined as in (4.9) with $\varepsilon=\nu_{j}$. Since, by Theorem 3.78 in [4], for every $\nu_{j} \in D_{0}$ the set $S_{f \infty\left(\cdot, \nu_{j}\right)}$ is $\sigma$-finite with respect to $\mathcal{H}^{N-1}$, we obtain $\left|D^{c} u\right|\left(N_{2}\right)=0$ (see Th. 3.92 (c) in [4]).

Finally, set $N_{0}=N_{1} \cup N_{2}$, where $\left|D^{c} u\right|\left(N_{0}\right)=0$ and choose $x_{0} \in\left(\Omega \backslash S_{u}\right) \backslash N_{0}$.

Then, taking into account (2.6), (2.8) and (4.10), we have

$$
\begin{aligned}
\frac{\mathrm{d} \bar{F}(u, \cdot)}{\mathrm{d}\left|D^{c} u\right|}\left(x_{0}\right)+1 & \leq \liminf _{h \rightarrow+\infty} \limsup _{\varepsilon \rightarrow 0^{+}} \frac{1}{h^{N-1} \varepsilon^{N} t_{\varepsilon}^{h}} \bar{F}_{1}\left(u_{\varepsilon}^{h}+\left\langle t_{\varepsilon}^{h} \nu, x-x_{0}\right\rangle, Q_{\nu}^{h}\left(x_{0}, \varepsilon\right)\right) \\
& \leq \liminf _{h \rightarrow+\infty} \limsup _{\varepsilon \rightarrow 0^{+}} f_{Q_{\nu}^{h}\left(x_{0}, \varepsilon\right)} \frac{f\left(x, t_{\varepsilon}^{h} \nu\right)}{t_{\varepsilon}^{h}} \mathrm{~d} x+1 \\
& \leq \liminf _{h \rightarrow+\infty} \limsup _{\varepsilon \rightarrow 0^{+}} f_{Q_{\nu}^{h}\left(x_{0}, \varepsilon\right)}\left(f^{\infty}(x, \nu)+\frac{f(x, 0)}{t_{\varepsilon}^{h}}\right) \mathrm{d} x+1 \\
& \leq \liminf _{h \rightarrow+\infty} \limsup _{\varepsilon \rightarrow 0^{+}} f_{Q_{\nu}^{h}\left(x_{0}, \varepsilon\right)} f^{\infty}(x, \nu) \mathrm{d} x+1,
\end{aligned}
$$

which implies

$$
\frac{\mathrm{d} \bar{F}(u, \cdot)}{\mathrm{d}\left|D^{c} u\right|}\left(x_{0}\right) \leq \liminf _{h \rightarrow+\infty} \limsup _{\varepsilon \rightarrow 0^{+}} f_{Q_{\nu}^{h}\left(x_{0}, \varepsilon\right)} f^{\infty}(x, \nu) \mathrm{d} x .
$$

Hence, in order to conclude, it is enough to prove that for all $h \in \mathbb{N}$

$$
\limsup _{\varepsilon \rightarrow 0^{+}} f_{Q_{\nu}^{h}\left(x_{0}, \varepsilon\right)} f^{\infty}(x, \nu) \mathrm{d} x \leq f^{\infty}\left(x_{0}, \nu\right) .
$$


Let $\left\{\nu_{j}\right\}$ be a sequence of directions contained in $D_{0}$ converging to $\nu$. By using the Lipschitz continuity of $f^{\infty}(x, \cdot)$ and recalling that $x_{0} \notin S_{f^{\infty}\left(\cdot, \nu_{j}\right)}$, it follows that for every $j \in \mathbb{N}$

$$
\begin{aligned}
\limsup _{\varepsilon \rightarrow 0^{+}} f_{Q_{\nu}^{h}\left(x_{0}, \varepsilon\right)} f^{\infty}(x, \nu) \mathrm{d} x & \leq \limsup _{\varepsilon \rightarrow 0^{+}} f_{Q_{\nu}^{h}\left(x_{0}, \varepsilon\right)} f^{\infty}\left(x, \nu_{j}\right) \mathrm{d} x+\Lambda \limsup _{\varepsilon \rightarrow 0^{+}} f_{Q_{\nu}^{h}\left(x_{0}, \varepsilon\right)}\left|\nu_{j}-\nu\right| \mathrm{d} x \\
& =f^{\infty}\left(x_{0}, \nu_{j}\right)+\Lambda\left|\nu_{j}-\nu\right| .
\end{aligned}
$$

Thus, letting $j \rightarrow+\infty,(4.13)$ is proved. Hence, the assertion follows.

Let us now describe the idea of the proof of $(J)$ which is really the new point in the whole relaxation argument. The main difficulty here, differently from the cases treated in [19] and [3], is due to the points where $f^{\infty}$ and $u$ both jump. To understand how we deal with this case, let us assume for simplicity that $f(x, \xi)=a(x) p(\xi)$, that $a$ and $u$ have the same jump set $\Gamma$ and that $\Gamma$ is a smooth manifold splitting $\Omega$ in two open sets $\Omega^{ \pm}$.

As in [19], our proof is based on a blow-up argument at any point $x_{0} \in \Gamma$ and on a formula which involves the jump function $w_{\nu}$ taking the two values $u^{ \pm}\left(x_{0}\right)$ and jumping along the tangent plane to $\Gamma$ at $x_{0}$. However this function would not work in our case. Instead, we have to replace it with the function $w_{\Gamma}$ jumping along $\Gamma$ itself. Moreover, in order to recover $a^{-}\left(x_{0}\right)$ we need to approximate $w_{\Gamma}$ by a sequence of functions $u^{n}$ such that $u^{n}=w_{\Gamma}$ on $\Omega^{+}$and smoothly interpolate between the two values $u^{ \pm}\left(x_{0}\right)$ on $\Omega^{-}$, if $\nu_{a}\left(x_{0}\right)$ points toward $\Omega^{+}$, and do the opposite if $\nu_{a}\left(x_{0}\right)$ points toward $\Omega^{-}$. Further complications come to play if $f$ cannot be split as a product, if the jump set of $f^{\infty}(\cdot, \xi)$ varies with $\xi$ and $\Gamma$ is not a smooth manifold.

Proposition 4.4. Let $f$ satisfy the assumptions of Theorem 4.2. Then, $(J)$ holds; i.e., for every $u \in \operatorname{BV}(\Omega)$,

$$
\frac{\mathrm{d} \bar{F}(u, \cdot)}{\mathrm{d} \mathcal{H}^{N-1}\left\lfloor J_{u}\right.}\left(x_{0}\right) \leq\left[u^{+}\left(x_{0}\right)-u^{-}\left(x_{0}\right)\right] f^{\infty}\left(x_{0}, \nu_{u}\left(x_{0}\right)\right) \quad \text { for } \mathcal{H}^{N-1} \text {-a.e. } x_{0} \in J_{u} .
$$

Proof. Let $u \in \operatorname{BV}(\Omega)$. Since $J_{u}$ is a countably $\mathcal{H}^{N-1}$-rectifiable set, we have that $J_{u}=\left(\cup K_{n}\right) \cup \widetilde{N}$, where $\mathcal{H}^{N-1}(\widetilde{N})=0, K_{n}$ are disjoint compact sets and $K_{n} \subseteq \Gamma_{n}$, where, for every $n \in \mathbb{N}, \Gamma_{n}$ is the graph of a $\mathcal{C}^{1}$-function. Moreover, by removing a set $N_{1} \subset J_{u}$ of zero $\mathcal{H}^{N-1}$-measure, we may assume that if $x \in K_{n} \backslash N_{1}$ for some $n$, then $\nu_{u}(x)$ is orthogonal to the tangent plane to $\Gamma_{n}$ at $x$.

To prove the assertion it is then enough to show that, for any $n$, (4.14) holds for $\mathcal{H}^{N-1}$-a.e. $x \in K_{n}$. To this aim, we fix $n$ and prove the assertion in $J_{u} \cap \Gamma_{n}$. However, in order to simplify the notation, from now on we drop the subscript $n$ by writing $\Gamma$ instead of $\Gamma_{n}$.

As in the proof of Proposition 4.3, let $D_{0}$ denote a countable dense subset of $\mathbb{S}^{N-1}$. By Theorem 3.7 of [7], for $\mathcal{H}^{N-1}$-almost every $x_{0} \in J_{u} \cap \Gamma$ we have

$$
\begin{aligned}
\frac{\mathrm{d} \bar{F}_{1}(u, \cdot)}{\mathrm{d} \mathcal{H}^{N-1}\left\lfloor J_{u}\right.}\left(x_{0}\right) & =\frac{\mathrm{d} \bar{F}(u, \cdot)}{\mathrm{d} \mathcal{H}^{N-1}\left\lfloor J_{u}\right.}\left(x_{0}\right)+\left|u^{+}\left(x_{0}\right)-u^{-}\left(x_{0}\right)\right| \\
& =\limsup _{\varepsilon \rightarrow 0^{+}} \frac{\inf \left\{\bar{F}_{1}\left(v, Q_{\nu}\left(x_{0}, \varepsilon\right)\right): v \in \operatorname{BV}\left(Q_{\nu}\left(x_{0}, \varepsilon\right)\right),\left.v\right|_{\partial Q_{\nu}\left(x_{0}, \varepsilon\right)}=w_{\nu}\right\}}{\varepsilon^{N-1}},
\end{aligned}
$$

where $\nu=\nu_{u}\left(x_{0}\right), Q_{\nu}\left(x_{0}, \varepsilon\right)=x_{0}+\varepsilon Q_{\nu}, Q_{\nu}$ is defined as in (4.11) with $h=1$, and $w_{\nu}$ is the jump function which takes the value $u^{+}\left(x_{0}\right)$ if $\left\langle x-x_{0}, \nu\right\rangle>0$ and $u^{-}\left(x_{0}\right)$ if $\left\langle x-x_{0}, \nu\right\rangle \leq 0$.

Let $N_{2} \subset J_{u}$, with $\mathcal{H}^{N-1}\left(N_{2}\right)=0$, be such that for every $x_{0} \in J_{u} \backslash N_{2}$ (4.15) holds.

Let $N_{3} \subset J_{u}$, with $\mathcal{H}^{N-1}\left(N_{3}\right)=0$, be such that for every $x_{0} \in\left(J_{u} \cap \Gamma\right) \backslash N_{3}$ Theorem 2.2 holds, with $g_{\Gamma}^{ \pm}$ replaced by $\left(f^{\infty}\left(\cdot, \nu_{j}\right)\right)_{\Gamma}^{ \pm}$, for every $\nu_{j} \in D_{0}$.

Let $N_{4} \subset J_{u}$, with $\mathcal{H}^{N-1}\left(N_{4}\right)=0$, be such that for every $x_{0} \in\left(J_{u} \cap \Gamma\right) \backslash N_{4}$ (2.4) and (2.5) hold, with $g_{\Gamma}^{ \pm}$ replaced by $\left(f^{\infty}\left(\cdot, \nu_{j}\right)\right)_{\Gamma}^{ \pm}$, for every $\nu_{j} \in D_{0}$. 
Let us now set

$$
N_{5}=\left(\bigcup_{j \in \mathbb{N}} S_{f^{\infty}\left(\cdot, \nu_{j}\right)} \backslash J_{f \infty\left(\cdot, \nu_{j}\right)}\right) \cap \Gamma .
$$

Then $\mathcal{H}^{N-1}\left(N_{5}\right)=0$. Let $N_{6} \subset J_{u}$, with $\mathcal{H}^{N-1}\left(N_{6}\right)=0$, be such that for every $x_{0} \in\left(J_{u} \cap J_{f \infty}\left(\cdot, \nu_{j}\right) \backslash N_{6}\right.$, $\nu_{u}\left(x_{0}\right)= \pm \nu_{f \infty\left(\cdot, \nu_{j}\right)}\left(x_{0}\right)$, for every $\nu_{j} \in D_{0}$. Finally, set $N_{0}=N_{1} \cup N_{2} \cup N_{3} \cup N_{4} \cup N_{5} \cup N_{6} \cup N_{7}$, where $N_{7}=\cup_{\nu_{j} \in D_{0}} N_{\nu_{j}}$ and $N_{\nu_{j}}$ is defined as in (4.9). Clearly $\mathcal{H}^{N-1}\left(N_{0}\right)=0$.

Let us fix $x_{0} \in\left(J_{u} \cap \Gamma\right) \backslash N_{0}$. For the sake of simplicity, we may assume $x_{0}=0$. Since $\nu=\nu_{u}(0)$ is orthogonal to $\Gamma$ at 0 , let us orient $\Gamma$ in such a way that $\nu_{\Gamma}(0)=\nu_{u}(0)$. Denote by $A$ an open neighborhood of the origin such that $\Gamma \cap A$ coincides with the graph of a $C^{1}$ function $\psi: \pi_{\nu^{\perp}}\left(\mathbb{R}^{N}\right) \rightarrow \mathbb{R}$ such that $\psi(0)=0$ and $\nabla_{x_{\nu}} \psi(0)=0$ and $A \backslash \Gamma$ is the union of two open sets $A^{ \pm}$, where the signs \pm are chosen so that, as in Remark $2.4, \nu_{\Gamma}(0)$ points toward $A^{+}$.

Let $\left\{\nu_{j}\right\}$ be a sequence of directions contained in $D_{0}$ converging to $\nu$. Notice that for $j$ sufficiently large there exist $C^{1}$ functions $\psi_{j}: \pi_{\nu_{j}^{\perp}}\left(\mathbb{R}^{N}\right) \rightarrow \mathbb{R}$ such that $\Gamma \cap A$ coincides with the graph of $\psi_{j}$. Finally denote by $w_{\Gamma}$ the jump function which takes the value $u^{ \pm}(0)$ in $A^{ \pm}$.

Let us fix $\delta>0$; then $(1-\delta) Q_{\nu} \subset Q_{\nu_{j}}$ for all $j$ sufficiently large. Let $\phi \in \mathcal{C}_{0}^{\infty}\left(Q_{\nu}\right)$ be a cut-off function such that $\phi(x)=1$ in $(1-\delta) Q_{\nu}$ and $|\nabla \phi| \leq c / \delta$. For every $\varepsilon>0$, set $\phi_{\varepsilon}(x)=\phi\left(\frac{x}{\varepsilon}\right)$, so that $\left|\nabla \phi_{\varepsilon}\right| \leq c / \varepsilon \delta$ and set $w_{\varepsilon, \nu, \Gamma}(x)=\phi_{\varepsilon}(x) w_{\Gamma}(x)+\left(1-\phi_{\varepsilon}(x)\right) w_{\nu}(x)$. We note that $w_{\varepsilon, \nu, \Gamma}$ satisfies the boundary condition $\left.w_{\varepsilon, \nu, \Gamma}\right|_{\partial\left(\varepsilon Q_{\nu}\right)}=w_{\nu}$, so that, by $(4.15)$, we obtain

$$
\frac{\mathrm{d} \bar{F}_{1}(u, \cdot)}{\mathrm{d} \mathcal{H}^{N-1}\left\lfloor J_{u}\right.}(0) \leq \limsup _{\varepsilon \rightarrow 0^{+}}\left[\frac{\bar{F}\left(w_{\varepsilon, \nu, \Gamma}, \varepsilon Q_{\nu}\right)}{\varepsilon^{N-1}}+\frac{\left|D w_{\varepsilon, \nu, \Gamma}\right|\left(\varepsilon Q_{\nu}\right)}{\varepsilon^{N-1}}\right] .
$$

Clearly, for every $\varepsilon>0$ and $j \in \mathbb{N}$ sufficiently large,

$$
\begin{aligned}
\frac{\left|D w_{\varepsilon, \nu, \Gamma}\right|\left(\varepsilon Q_{\nu}\right)}{\varepsilon^{N-1}} & \leq \frac{1}{\varepsilon^{N-1}}\left[\int_{\varepsilon Q_{\nu}}\left|\nabla \phi_{\varepsilon}\right|\left|w_{\Gamma}-w_{\nu}\right| \mathrm{d} x+\left|D w_{\Gamma}\right|\left(\varepsilon Q_{\nu}\right)+\left|D w_{\nu}\right|\left(\varepsilon Q_{\nu} \backslash \varepsilon(1-\delta) Q_{\nu}\right)\right] \\
& \leq \frac{c}{\varepsilon^{N} \delta} \int_{\varepsilon Q_{\nu}}\left|w_{\Gamma}-w_{\nu}\right| \mathrm{d} x+\frac{\left|u^{+}-u^{-}\right|}{\varepsilon^{N-1}} \mathcal{H}^{N-1}\left(\Gamma \cap \varepsilon Q_{\nu}\right)+c \delta \\
& \leq \frac{c}{\varepsilon^{N} \delta} \int_{\varepsilon Q_{\nu}^{\perp}}\left|\psi\left(x_{\nu}^{\perp}\right)\right| \mathrm{d} x_{\nu}^{\perp}+\frac{\left|u^{+}-u^{-}\right|}{\varepsilon^{N-1}} \int_{\varepsilon Q_{\nu}^{\perp}} \sqrt{1+\left|\nabla_{x_{\nu}} \psi\left(x_{\nu}^{\perp}\right)\right|^{2}} \mathrm{~d} x_{\nu}^{\perp}+c \delta \\
& \leq \frac{c}{\delta}\|\nabla \psi\|_{L^{\infty}\left(\varepsilon Q_{\nu}^{\perp}\right)}+\left|u^{+}-u^{-}\right| \int_{\varepsilon Q_{\nu}^{\perp}} \sqrt{1+\left|\nabla_{x_{\nu}^{\perp}} \psi\left(x_{\nu}^{\perp}\right)\right|^{2}} \mathrm{~d} x_{\nu}^{\perp}+c \delta,
\end{aligned}
$$

where $u^{+}, u^{-}$stands for $u^{+}(0), u^{-}(0)$, respectively, and $Q_{\nu}^{\perp}=\pi_{\nu^{\perp}}\left(Q_{\nu}\right)$. Letting $\varepsilon \rightarrow 0^{+}$and recalling that $\nabla_{x_{\nu}^{\perp}} \psi(0)=0$, we obtain

$$
\limsup _{\varepsilon \rightarrow 0^{+}} \frac{\left|D w_{\varepsilon, \nu, \Gamma}\right|\left(\varepsilon Q_{\nu}\right)}{\varepsilon^{N-1}} \leq\left|u^{+}-u^{-}\right|+c \delta .
$$

Let us now fix $j \in \mathbb{N}$ and assume, in order to fix the ideas, that $0 \in J_{f \infty\left(\cdot, \nu_{j}\right)}$ and $\nu_{f} \infty_{\left(\cdot, \nu_{j}\right)}(0)=\nu_{u}(0)$. We approximate $w_{\varepsilon, \nu, \Gamma}$ with the functions $u_{\varepsilon, \nu, j}^{n}(x)=\phi_{\varepsilon}(x) u_{\varepsilon, j}^{n}(x)+\left(1-\phi_{\varepsilon}(x)\right) u_{\varepsilon, \nu}^{n}(x)$, where

$$
u_{\varepsilon, \nu}^{n}(x):= \begin{cases}u^{+} & \text {if }\langle x, \nu\rangle \geq 0, \\ \left(u^{+}-u^{-}\right) \frac{n}{\varepsilon}\langle x, \nu\rangle+u^{+} & \text {if }-\varepsilon / n \leq\langle x, \nu\rangle \leq 0, \\ u^{-} & \text {if }\langle x, \nu\rangle \leq-\varepsilon / n,\end{cases}
$$


and

$$
u_{\varepsilon, j}^{n}(x):= \begin{cases}u^{+} & \text {if } x_{\nu_{j}} \geq \psi_{j}\left(x_{\nu_{j}}^{\perp}\right) \\ \left(u^{+}-u^{-}\right) \frac{n}{\varepsilon}\left[x_{\nu_{j}}-\psi_{j}\left(x_{\nu_{j}}^{\perp}\right)\right]+u^{+} & \text {if } \psi_{j}\left(x_{\nu_{j}}^{\perp}\right)-\varepsilon / n \leq x_{\nu_{j}} \leq \psi_{j}\left(x_{\nu_{j}}^{\perp}\right) \\ u^{-} & \text {if } x_{\nu_{j}} \leq \psi_{j}\left(x_{\nu_{j}}^{\perp}\right)-\varepsilon / n\end{cases}
$$

It will be clear from the rest of the proof that, when $0 \in J_{f^{\infty}\left(\cdot, \nu_{j}\right)}$ and $\nu_{f \infty}\left(\cdot, \nu_{j}\right)(0)=-\nu_{u}(0)$, the argument below still works if we define

$$
u_{\varepsilon, j}^{n}(x):= \begin{cases}u^{+} & \text {if } x_{\nu_{j}} \geq \psi_{j}\left(x_{\nu_{j}}^{\perp}\right)+\varepsilon / n \\ \left(u^{+}-u^{-}\right) \frac{n}{\varepsilon}\left[x_{\nu_{j}}-\psi_{j}\left(x_{\nu_{j}}^{\perp}\right)\right]+u^{-} & \text {if } \psi_{j}\left(x_{\nu_{j}}^{\perp}\right) \leq x_{\nu_{j}} \leq \psi_{j}\left(x_{\nu_{j}}^{\perp}\right)+\varepsilon / n \\ u^{-} & \text {if } x_{\nu_{j}} \leq \psi_{j}\left(x_{\nu_{j}}^{\perp}\right)\end{cases}
$$

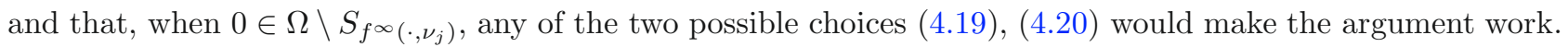
Notice, however, that in all three cases in the definition of $u_{\varepsilon, \nu}^{n}$ remains unchanged.

The functions $u_{\varepsilon, \nu}^{n}, u_{\varepsilon, j}^{n}$ belong to $W^{1,1}\left(\varepsilon Q_{\nu}\right)$ and $\left\|u_{\varepsilon, \nu}^{n}-w_{\nu}\right\|_{L^{1}\left(\varepsilon Q_{\nu}\right)} \rightarrow 0,\left\|u_{\varepsilon, j}^{n}-w_{\Gamma}\right\|_{L^{1}\left(\varepsilon Q_{\nu}\right)} \rightarrow 0$, hence $\left\|u_{\varepsilon, \nu, j}^{n}-w_{\varepsilon, \nu, \Gamma}\right\|_{L^{1}\left(\varepsilon Q_{\nu}\right)} \rightarrow 0$, as $n \rightarrow+\infty$. Therefore, by the lower semicontinuity of $\bar{F},(2.8)$, (2.6), we have that for all $\varepsilon>0$ and $j$ sufficiently large,

$$
\begin{aligned}
\frac{\bar{F}\left(w_{\varepsilon, \nu, \Gamma}, \varepsilon Q_{\nu}\right)}{\varepsilon^{N-1}} \leq & \liminf _{n \rightarrow+\infty} \frac{1}{\varepsilon^{N-1}} \int_{\varepsilon Q_{\nu}} f\left(x, \nabla u_{\varepsilon, \nu, j}^{n}\right) \mathrm{d} x \\
\leq & \liminf _{n \rightarrow+\infty} \frac{1}{\varepsilon^{N-1}}\left[\int_{\varepsilon(1-\delta) Q_{\nu}} f\left(x, \nabla u_{\varepsilon, j}^{n}\right) \mathrm{d} x+\Lambda \int_{\varepsilon Q_{\nu}}\left[\left|\nabla \phi_{\varepsilon}\right|\left|u_{\varepsilon, j}^{n}-u_{\varepsilon, \nu}^{n}\right|+1\right] \mathrm{d} x\right. \\
& \left.+\Lambda \int_{\varepsilon Q_{\nu} \backslash \varepsilon(1-\delta) Q_{\nu}}\left(\phi_{\varepsilon}\left|\nabla u_{\varepsilon, j}^{n}\right|+\left(1-\phi_{\varepsilon}\right)\left|\nabla u_{\varepsilon, \nu}^{n}\right|\right) \mathrm{d} x\right] \\
\leq & \liminf _{n \rightarrow+\infty}\left[f_{\varepsilon Q_{\nu_{j}}} \varepsilon f\left(x, \nabla u_{\varepsilon, j}^{n}\right) \mathrm{d} x+\frac{c}{\delta} f_{\varepsilon Q_{\nu}}\left|u_{\varepsilon, j}^{n}-u_{\varepsilon, \nu}^{n}\right| \mathrm{d} x\right]+c \varepsilon+c \delta \\
\leq & \liminf _{n \rightarrow+\infty} \int_{\varepsilon Q_{\nu_{j}}}\left[f^{\infty}\left(x, \varepsilon \nabla u_{\varepsilon, j}^{n}\right)+\varepsilon f(x, 0)\right] \mathrm{d} x+\frac{c}{\delta} f_{\varepsilon Q_{\nu}}\left|w_{\Gamma}-w_{\nu}\right| \mathrm{d} x+c \varepsilon+c \delta \\
\leq & \liminf _{n \rightarrow+\infty} f_{\varepsilon Q_{\nu_{j}}} f^{\infty}\left(x, \varepsilon \nabla u_{\varepsilon, j}^{n}\right) \mathrm{d} x+\frac{c}{\delta}\left\|\nabla_{x_{\nu}^{\perp}} \psi\right\|_{L^{\infty}\left(\varepsilon Q_{\nu}^{\perp}\right)}+c \varepsilon+c \delta,
\end{aligned}
$$

where the last inequality $f_{\varepsilon Q_{\nu}}\left|w_{\Gamma}-w_{\nu}\right| \mathrm{d} x \leq c\left\|\nabla_{x_{\nu}^{\perp}} \psi\right\|_{L^{\infty}\left(\varepsilon Q_{\nu}\right)}$ is obtained as in (4.17). Letting $\varepsilon \rightarrow 0^{+}$, we obtain

$$
\limsup _{\varepsilon \rightarrow 0^{+}} \frac{\bar{F}\left(w_{\varepsilon, \nu, \Gamma}, \varepsilon Q_{\nu}\right)}{\varepsilon^{N-1}} \leq \limsup _{\varepsilon \rightarrow 0^{+}} \liminf _{n \rightarrow+\infty} f_{\varepsilon Q_{\nu_{j}}} f^{\infty}\left(x, \varepsilon \nabla u_{\varepsilon, j}^{n}\right) \mathrm{d} x+c \delta
$$


where we have used the equality $\nabla_{x_{\nu}^{\perp}} \psi(0)=0$. Moreover, by the Lipschitz continuity of $f^{\infty}(x, \cdot)$, we get, setting $\widehat{\psi}_{j}(x)=\psi_{j}\left(x-\left\langle x, \nu_{j}\right\rangle \nu_{j}\right)$,

$$
\begin{aligned}
& \limsup _{\varepsilon \rightarrow 0^{+}} \liminf _{n \rightarrow+\infty} f_{\varepsilon Q_{\nu_{j}}} f^{\infty}\left(x, \varepsilon \nabla u_{\varepsilon, j}^{n}\right) \mathrm{d} x \\
& \leq\left(u^{+}-u^{-}\right) \limsup _{\varepsilon \rightarrow 0^{+}} \liminf _{n \rightarrow+\infty} f_{\varepsilon Q_{\nu_{j}}^{\perp}}\left[\frac{n}{\varepsilon} \int_{\psi_{j}\left(x_{\nu_{j}}^{\perp}\right)-\varepsilon / n}^{\psi_{j}\left(x_{\nu_{j}}^{\perp}\right)} f^{\infty}\left(x_{\nu_{j}}^{\perp}, x_{\nu_{j}}, \nu_{j}-\left(\nabla_{x} \widehat{\psi}_{j}\right)\left(x_{\nu_{j}}^{\perp}, x_{\nu_{j}}\right)\right) \mathrm{d} x_{\nu_{j}}\right] \mathrm{d} x_{\nu_{j}}^{\perp} \\
& \leq\left(u^{+}-u^{-}\right) \limsup _{\varepsilon \rightarrow 0^{+}} \liminf _{n \rightarrow+\infty} f_{\varepsilon Q_{\nu_{j}}^{\perp}}\left[\frac{n}{\varepsilon} \int_{\psi_{j}\left(x_{\nu_{j}}^{\perp}\right)-\varepsilon / n}^{\psi_{j}\left(x_{\nu_{j}}^{\perp}\right)} f^{\infty}\left(x_{\nu_{j}}^{\perp}, x_{\nu_{j}}, \nu_{j}\right) \mathrm{d} x_{\nu_{j}}\right] \mathrm{d} x_{\nu_{j}}^{\perp} \\
& +c \limsup _{\varepsilon \rightarrow 0^{+}} f_{\varepsilon Q_{\nu_{j}}^{\perp}}\left|\nabla_{x_{\nu_{j}}^{\perp}} \psi_{j}\left(x_{\nu_{j}}^{\perp}\right)\right| \mathrm{d} x_{\nu_{j}}^{\perp} \\
& \leq\left(u^{+}-u^{-}\right) \limsup _{\varepsilon \rightarrow 0^{+}} \liminf _{n \rightarrow+\infty} \int_{\varepsilon Q_{\nu_{j}}^{\perp}}\left[\frac{n}{\varepsilon} \int_{\psi_{j}\left(x_{\nu_{j}}^{\perp}\right)-\varepsilon / n}^{\psi_{j}\left(x_{\nu_{j}}^{\perp}\right)} f^{\infty}\left(x_{\nu_{j}}^{\perp}, x_{\nu_{j}}, \nu_{j}\right) \mathrm{d} x_{\nu_{j}}\right] \mathrm{d} x_{\nu_{j}}^{\perp} \\
& \leq\left(u^{+}-u^{-}\right) \limsup _{\varepsilon \rightarrow 0^{+}} \liminf _{n \rightarrow+\infty} f_{\varepsilon Q_{\nu_{\nu_{j}}}^{\perp}}\left[\frac{n}{\varepsilon} \int_{\psi_{j}\left(x_{\nu_{j}}\right)-\varepsilon / n}^{\psi_{j}\left(x_{\nu_{j}}^{\perp}\right)} f^{\infty}\left(x_{\nu_{j}}^{\perp}, x_{\nu_{j}}, \nu_{x_{j}}\right) \mathrm{d} x_{\nu_{j}}\right] \mathrm{d} x_{\nu_{j}}^{\perp} \|_{L^{\infty}\left(\varepsilon Q_{\nu_{j}}\right)}^{\perp}+c\left|\nabla_{x_{\nu_{j}}^{\perp}} \psi_{j}(0)\right|
\end{aligned}
$$

where $Q_{\nu_{j}}^{\perp}=\pi_{\nu_{j}^{\perp}}\left(Q_{\nu_{j}}\right)$. Notice that, by Lemma 2.1 we have that, for $\mathcal{H}^{N-1}$-a.e. $x_{\nu_{j}}^{\perp} \in \varepsilon Q_{\nu_{j}}^{\perp}$,

$$
\begin{aligned}
& \lim _{n \rightarrow+\infty} \frac{n}{\varepsilon} \int_{\psi_{j}\left(x_{\nu_{j}}^{\perp}\right)-\varepsilon / n}^{\psi_{j}\left(x_{\nu_{j}}^{\perp}\right)} f^{\infty}\left(x_{\nu_{j}}^{\perp}, x_{\nu_{j}}, \nu_{j}\right) \mathrm{d} x_{\nu_{j}} \\
& = \begin{cases}\widetilde{f^{\infty}}\left(x_{\nu_{j}}^{\perp}, \psi_{j}\left(x_{\nu_{j}}^{\perp}\right), \nu_{j}\right) & \text { if }\left(x_{\nu_{j}}^{\perp}, \psi_{j}\left(x_{\nu_{j}}^{\perp}\right) \in \Omega \backslash J_{f \infty}\left(\cdot, \nu_{j}\right)\right. \\
\left(f^{\infty}\right)^{-}\left(x_{\nu_{j}}^{\perp}, \psi_{j}\left(x_{\nu_{j}}^{\perp}\right), \nu_{j}\right) & \text { if }\left(x_{\nu_{j}}^{\perp}, \psi_{j}\left(x_{\nu_{j}}^{\perp}\right) \in J_{f^{\infty}\left(\cdot, \nu_{j}\right)},\left\langle\nu_{j}, \nu_{f \infty}\left(\cdot, \nu_{j}\right)\right.\right. \\
\left(f^{\infty}\right)^{+}\left(x_{\nu_{j}}^{\perp}, \psi_{j}\left(x_{\nu_{j}}^{\perp}, \nu_{j}\right)\right. & \text { if } \left.\left.\left.\left.\left(x_{\nu_{j}}^{\perp}, \psi_{j}\left(x_{\nu_{j}}^{\perp}\right)\right) \in J_{f_{j}^{\infty}\left(\cdot, \nu_{j}\right)}^{\perp}\right)\right\rangle \nu_{j}, \nu_{f^{\infty}\left(\cdot, \nu_{j}\right)}\right)\right\rangle=0 \\
\left.\left.x_{\nu_{j}}, \psi_{j}\left(x_{\nu_{j}}^{\perp}\right)\right)\right\rangle 0 .\end{cases}
\end{aligned}
$$

In any case, (2.3) and the assumption that $\nu$ and $\nu_{\Gamma}(x)$ have the same orientation, yield

$$
\lim _{n \rightarrow+\infty} \frac{n}{\varepsilon} \int_{\psi_{j}\left(x_{\nu_{j}}^{\perp}\right)-\varepsilon / n}^{\psi_{j}\left(x_{\nu_{j}}^{\perp}\right)} f^{\infty}\left(x_{\nu_{j}}^{\perp}, x_{\nu_{j}}, \nu_{j}\right) \mathrm{d} x_{\nu_{j}}=\left(f^{\infty}\left(\cdot, \nu_{j}\right)\right)_{\Gamma}^{-}\left(x_{\nu_{j}}^{\perp}, \psi_{j}\left(x_{\nu_{j}}^{\perp}\right)\right)
$$

for $\mathcal{H}^{N-1}$-a.e. $x_{\nu_{j}}^{\perp} \in \varepsilon Q_{\nu_{j}}^{\perp}$. Therefore, by the dominated convergence theorem, we get

$$
\begin{aligned}
\limsup _{\varepsilon \rightarrow 0^{+}} \liminf _{n \rightarrow+\infty} f_{\varepsilon Q_{\nu_{j}}^{\perp}}\left(u^{+}-u^{-}\right)\left[\frac{n}{\varepsilon} \int_{\psi_{j}\left(x_{\nu_{j}}^{\perp}\right)-\varepsilon / n}^{\psi_{j}\left(x_{\nu_{j}}^{\perp}\right)} f^{\infty}\left(x_{\nu_{j}}^{\perp}, x_{\nu_{j}}, \nu_{j}\right) \mathrm{d} x_{\nu_{j}}\right] \mathrm{d} x_{\nu_{j}}^{\perp} \\
\quad=\limsup _{\varepsilon \rightarrow 0^{+}} f_{\varepsilon Q_{\nu_{j}}^{\perp}}\left(u^{+}-u^{-}\right) \lim _{n \rightarrow+\infty}\left[\frac{n}{\varepsilon} \int_{\psi_{j}\left(x_{\nu_{j}}^{\perp}\right)-\varepsilon / n}^{\psi_{j}\left(x_{\nu_{j}}^{\perp}\right)} f^{\infty}\left(x_{\nu_{j}}^{\perp}, x_{\nu_{j}}, \nu_{j}\right) \mathrm{d} x_{\nu_{j}}\right] \mathrm{d} x_{\nu_{j}}^{\perp} \\
=\limsup _{\varepsilon \rightarrow 0^{+}} f_{\varepsilon Q_{\nu_{j}}^{\perp}}\left(u^{+}-u^{-}\right)\left(f^{\infty}\left(\cdot, \nu_{j}\right)\right)_{\Gamma}^{-}\left(x_{\nu_{j}}^{\perp}, \psi_{j}\left(x_{\nu_{j}}^{\perp}\right)\right) \mathrm{d} x_{\nu_{j}}^{\perp} .
\end{aligned}
$$


Recalling that $\Gamma \cap \varepsilon Q_{\nu_{j}}$ can be parametrized by $\left(x_{\nu_{j}}^{\perp}, \psi_{j}\left(x_{\nu_{j}}^{\perp}\right)\right), x_{\nu_{j}}^{\perp} \in \varepsilon Q_{\nu_{j}}^{\perp}$, that 0 is a Lebesgue point for $\left(f^{\infty}\left(\cdot, \nu_{j}\right)\right)_{\Gamma}^{-}$, that $0 \in J_{f \infty}\left(\cdot, \nu_{j}\right)$ and $\nu_{f}{ }_{\left(\cdot, \nu_{j}\right)}(0)=\nu_{u}(0)=\nu_{\Gamma}(0)$, we obtain, recalling $(2.2)$ and $(2.3)$,

$$
\begin{aligned}
& \limsup _{\varepsilon \rightarrow 0^{+}} \int_{\varepsilon Q_{\nu_{j}}^{\perp}}\left(u^{+}-u^{-}\right)\left(f^{\infty}\left(\cdot, \nu_{j}\right)\right)_{\Gamma}^{-}\left(x_{\nu_{j}}^{\perp}, \psi_{j}\left(x_{\nu_{j}}^{\perp}\right)\right) \mathrm{d} x_{\nu_{j}}^{\perp} \\
& \quad=\limsup _{\varepsilon \rightarrow 0^{+}} \frac{1}{\varepsilon^{N-1}} \int_{\varepsilon Q_{\nu_{j}}^{\perp}}\left(u^{+}-u^{-}\right)\left[\frac{\left(f^{\infty}\left(\cdot, \nu_{j}\right)\right)_{\Gamma}^{-}\left(x_{\nu_{j}}^{\perp}, \psi_{j}\left(x_{\nu_{j}}^{\perp}\right)\right)}{\sqrt{1+\left|\nabla_{x_{\nu_{j}}} \psi_{j}\right|^{2}}} \sqrt{1+\left|\nabla_{x_{\nu_{j}}} \psi_{j}\right|^{2}}\right] \mathrm{d} x_{\nu_{j}}^{\perp} \\
& \quad=\lim _{\varepsilon \rightarrow 0^{+}}\left(f_{\varepsilon Q_{\nu_{j}}^{\perp}} \sqrt{1+\left|\nabla_{x_{\nu_{j}}^{\perp}} \psi_{j}\right|^{2}} \mathrm{~d} x_{\nu_{j}}^{\perp}\right)\left(f_{\Gamma \cap \varepsilon Q_{\nu_{j}}}\left(u^{+}-u^{-}\right) \frac{\left(f^{\infty}\left(\cdot, \nu_{j}\right)\right)_{\Gamma}^{-}(x)}{\sqrt{1+\left|\nabla_{x_{\nu_{j}}^{\perp}} \psi_{j}\right|^{2}}} \mathrm{~d} \mathcal{H}^{N-1}(x)\right) \\
& =\left(\sqrt{1+\left|\nabla_{x_{\nu_{j}}} \psi_{j}(0)\right|^{2}}\right)\left(\left(u^{+}-u^{-}\right) \frac{\left(f^{\infty}\left(\cdot, \nu_{j}\right)\right)_{\Gamma}^{-}(0)}{\sqrt{1+\left|\nabla_{x_{\nu_{j}}} \psi_{j}(0)\right|^{2}}}\right) \\
& =\left(u^{+}-u^{-}\right)\left(f^{\infty}\right)^{-}\left(0, \nu_{j}\right)=\left(u^{+}-u^{-}\right) f^{\infty}\left(0, \nu_{j}\right),
\end{aligned}
$$

where the last equality follows from the fact that $0 \notin \cup_{j} N_{\nu_{j}}$ and each $N_{\nu_{j}}$ is defined as in (4.9).

By (4.15), (4.16), (4.18), (4.21)-(4.24), we obtain

$$
\frac{\mathrm{d} \bar{F}(u, \cdot)}{\mathrm{d} \mathcal{H}^{N-1}\left\lfloor J_{u}\right.}(0)+\left|u^{+}-u^{-}\right| \leq\left(u^{+}-u^{-}\right) f^{\infty}\left(0, \nu_{j}\right)+c\left|\nabla_{x_{\nu_{j}}} \psi_{j}(0)\right|+c \delta+\left|u^{+}-u^{-}\right| .
$$

Therefore, taking into account the Lipschitz continuity of $f^{\infty}$ with respect to the last variable and recalling that $\nabla_{x_{\nu_{j}}} \psi_{j}(0) \rightarrow \nabla_{x_{\nu}} \psi(0)=0$, letting first $j \rightarrow+\infty$ and then $\delta \rightarrow 0^{+}$, we get

$$
\frac{\mathrm{d} \bar{F}(u, \cdot)}{\mathrm{d} \mathcal{H}^{N-1}\left\lfloor J_{u}\right.}(0)+\left|u^{+}-u^{-}\right| \leq\left(u^{+}-u^{-}\right) f^{\infty}\left(0, \nu_{u}\right)+\left|u^{+}-u^{-}\right| .
$$

Hence, the assertion follows.

We are now in position to give the proof of Theorem 4.2.

Proof of Theorem 4.2. Taking into account [19], Theorem 1.3, part (i), and Propositions 4.3 and 4.4, we obtain the assertion for any function $u \in \operatorname{BV}(\Omega)$ and any $A \in \mathcal{A}(\Omega)$.

\subsection{The proof of the relaxation result}

Thanks to the results obtained in previous sections, we are now able to give the proof of the relaxation Theorem 1.1.

Proof of Theorem 1.1. Let us start by observing that since for every $\xi \in \mathbb{R}^{N}$ the functions $f(\cdot, \xi)$ and $f^{-}(\cdot, \xi)$ agree $\mathcal{L}^{N}$-a.e. in $\Omega$, then it is easily checked that for every summable function $z: \Omega \rightarrow \mathbb{R}^{N}$

$$
\int_{\Omega} f(x, z(x)) \mathrm{d} x=\int_{\Omega} f^{-}(x, z(x)) \mathrm{d} x .
$$

Therefore, denoting by $\mathcal{F}$ the functional in (1.7), we may replace $f$ by $f^{-}$in the first integral without affecting the value of the functional. 
By Theorem $2.6 f^{-}(\cdot, \xi)$ is $C_{1}$-quasi lower semicontinuous for all $\xi$. Therefore inequality $\bar{F}(u) \geq \mathcal{F}(u)$ follows at once by applying Theorem 3.1 to $a^{-}(x) p(\xi)$ (if (1.4) holds) or Theorem 3.6 to $f^{-}(x, \xi)$ (if (1.5) or (1.6) are in force).

The opposite inequality $\mathcal{F}(u) \geq \bar{F}(u)$ follows by applying Theorem 4.2 to $f^{-}$and observing that (4.9) is always satisfied if (1.4) or (1.5) holds.

\section{REFERENCES}

[1] M. Amar, and G. Bellettini, A notion of total variation depending on a metric with discontinuous coefficients. Ann. Inst. Henri Poincaré 11 (1994) 91-133.

[2] M. Amar and V. De Cicco, Relaxation in $B V$ for a class of functionals without continuity assumptions. NoDEA Nonlinear Differential Equations Appl. (to appear).

[3] M. Amar, V. De Cicco and N. Fusco, A relaxation result in BV for integral functionals with discontinuous integrands. ESAIM: COCV 13 (2007) 396-412.

[4] L. Ambrosio, N. Fusco and D. Pallara, Functions of bounded variation and free discontinuity problems. Oxford University Press, New York (2000).

[5] G. Anzellotti, G. Buttazzo and G. Dal Maso, Dirichlet problem for demi-coercive functionals. Nonlinear Anal. 10 (1986) 603-613.

[6] G. Bouchitté and M. Valadier, Integral representation of convex functionals on a space of measures. J. Funct. Anal. 80 (1988) 398-420.

[7] G. Bouchitté, I. Fonseca and L. Mascarenhas, A global method for relaxation. Arch. Rat. Mech. Anal. 145 (1998) 51-98.

[8] G. Buttazzo, Semicontinuity, Relaxation and Integral Representation Problems in the Calculus of Variations. Pitman Res. Notes in Math., Longman, Harlow (1989).

[9] M. Carriero, G. Dal Maso, A. Leaci and E. Pascali, Relaxation of the non-parametric Plateau problem with an obstacle. J. Math. Pures Appl. 67 (1988) 359-396.

[10] G. Dal Maso, Integral representation on $B V(\Omega)$ of $\Gamma$-limits of variational integrals. Manuscripta Math. 30 (1980) $387-416$.

[11] G. Dal Maso, On the integral representation of certain local functionals. Ricerche di Matematica 32 (1983) 85-113.

[12] G. Dal Maso, An Introduction to $\Gamma$-convergence. Birkhäuser, Boston (1993).

[13] V. De Cicco and G. Leoni, A chain rule in $L^{1}(\operatorname{div} ; \Omega)$ and its applications to lower semicontinuity. Calc. Var. Partial Differential Equations 19 (2004) 23-51.

[14] V. De Cicco, N. Fusco and A. Verde, On $L^{1}$-lower semicontinuity in $B V(\Omega)$. J. Convex Analysis 12 (2005) $173-185$.

[15] V. De Cicco, N. Fusco and A. Verde, A chain rule formula in $B V(\Omega)$ and its applications to lower semicontinuity. Calc. Var. Partial Differential Equations 28 (2007) 427-447.

[16] E. De Giorgi and T. Franzoni, Su un tipo di convergenza variazionale. Atti Accad. Naz. Lincei Rend. Cl. Sci. Fis. Mat. Natur. 58 (1975) 842-850.

[17] E. De Giorgi and T. Franzoni, Su un tipo di convergenza variazionale. Rend. Sem. Mat. Brescia 3 (1979) 63-101.

[18] H. Federer and W.P. Ziemer, The Lebesgue set of a function whose distribution derivatives are $p$-th power summable. Indiana Un. Math. J. 22 (1972) 139-158.

[19] I. Fonseca and G. Leoni, On lower semicontinuity and relaxation. Proc. Royal Soc. Edinb., Sect. A, Math. 131 (2001) $519-565$.

[20] I. Fonseca and S. Müller, Quasi-convex integrands and lower semicontinuity in L $L^{1}$. SIAM J. Math. Anal. 23 (1992) 1081-1098.

[21] I. Fonseca and S. Müller, Relaxation of quasiconvex functionals in $\operatorname{BV}\left(\Omega, \mathbb{R}^{p}\right)$ for integrands $f(x, u, \nabla u)$. Arch. Rat. Mech. Anal. 123 (1993) 1-49.

[22] N. Fusco, F. Giannetti and A. Verde, A remark on the $L^{1}$-lower semicontinuity for integral functionals in BV. Manuscripta Math. 112 (2003) 313-323.

[23] N. Fusco, M. Gori and F. Maggi, A remark on Serrin's Theorem. NoDEA Nonlinear Differential Equations Appl. 13 (2006) $425-433$.

[24] M. Gori and F. Maggi, The common root of the geometric conditions in Serrin's semicontinuity theorem. Ann. Mat. Pura Appl. 184 (2005) 95-114.

[25] M. Gori, F. Maggi and P. Marcellini, On some sharp conditions for lower semicontinuity in L1. Diff. Int. Eq. 16 (2003) 51-76.

[26] F. Maggi, On the relaxation on BV of certain non coercive integral functionals. J. Convex Anal. 10 (2003) 477-489.

[27] M. Miranda, Superfici cartesiane generalizzate ed insiemi di perimetro localmente finito sui prodotti cartesiani. Ann. Scuola Norm. Sup. Pisa 18 (1964) 515-542.

[28] Y.G. Reshetnyak, Weak convergence of completely additive vector functions on a set. Siberian Math. J. 9 (1968) $1039-1045$. 